\author{
Grégory DEBOUT, \\ Yann LE JEUNE, \\ Hélène DJÉMA, \\ Olivier BIGNON, \\ Marie-Aline CHARIER \\ et Caroline PESCHAUX
}

\section{La découverte du gisement de la Haye aux Mureaux (Yvelines) et ses implications sur la connaissance du peuplement magdalénien d'Île-de-France}

Résumé

Une opération d'archéologie préventive réalisée durant l'été 2009 dans le département des Yvelines, au cour du territoire Seine-Aval, a permis de repérer une unité d'occupation très bien conservée qui est rapportée à la phase supérieure du Magdalénien. Elle a été découverte dans une couche d'alluvions limono-sableuses qui s'est révélée un contexte particulièrement favorable à la conservation des vestiges. Actuellement, seuls une dizaine de mètres carrés ont été fouillées, mais les sondages réalisés dans les environs immédiats indiquent qu'elle se développe sur une surface d'environ 200 mètres carrés. À l'exception des éléments les plus fragiles qui ont été prélevés, les vestiges ont été conservés in situ pour une fouille ultérieure. Ils se composent principalement d'ossements et de ramures de renne, d'outils et de déchets de taille en silex, d'une pointe de sagaie ainsi que d'un probable élément de parure. L'ensemble se répartit de manière structurée autour d'un foyer en cuvette aménagé au moyen de blocs rocheux et à proximité duquel s'étend une nappe d'ocre rouge. La faible densité des vestiges évoque un séjour plutôt bref que les premiers indices de saisonnalité tendent à placer à l'automne. L'équipement technique et la structuration de l'espace habité permettent d'établir de nombreuses comparaisons avec quelques autres gisements magdaléniens de plein air du Bassin parisien. Par ailleurs, nous suggérons provisoirement une possible relation de contemporanéité entre cette unité d'occupation et deux autres ensembles (un crâne de cheval et une structure pierreuse) découverts à quelques centaines de mètres de distance dans une position stratigraphique analogue. Les aspects chronologiques sont d'ores et déjà documentés au moyen de datations radiocarbone obtenues sur os. Nous fondons aussi de nombreux espoirs sur les résultats d'analyses OSL (Optically Stimulated Luminescence) en cours qui pourraient permettre un calage plus précis de l'occupation au sein de l'une des chronozones du Tardiglaciaire weichsélien.

Mots-clés

Magdalénien supérieur, Bassin parisien, chronologie, géoarchéologie, organisation spatiale. 


\begin{abstract}
During the summer of 2009, an important archaeological campaign was carried out in the heart of the downstream Seine region. It allowed us to locate a well-preserved occupation site attributed to the Upper Magdalenian. The operation concerns a surface area of 103 hectares located from the left bank of the Seine along the edge of the river and slightly downstream, to the most concave part of a meander which gives a strongly asymmetrical aspect to the bottom of the valley. The Magdalenian occupation was discovered in an alluvial sandy silt layer which provides a favourable context for the preservation of archaeological remains. The occupation is located on the edge of a former channel, probably restored during the Late Glacial. A gravel ridge has protected the deposits from the Holocene alluvial erosion.

At present, only a surface of about 10 square meters in the main find concentration has been uncovered, but a quantity of test pits show that the archaeological layer extends over nearly 200 square meters. We provisionally suggest a possible relationship of contemporaneity between this occupation unit and two other sets of remains (a horse skull and a stony structure) discovered a few hundred metres away, in a similar stratigraphic position. With the exception of a few fragile remains that were removed during the field work, the other objects have been left in situ and the entire occupation site has been preserved for later excavations. The archaeological remains are mainly composed of reindeer bones and antlers, fint tools, backed bladelets and lithic remains, an antler point as well as a probable shell adornment.
\end{abstract}

More than two hundred flint artefacts were removed, among which we found two burins. The raw materials exploited by the Magdalenians consisted mainly of a flint of the Late Cretaceous epoch, probably stemming from the Campanian and Santonian stages. Furthermore, the presence of a tertiary material has been detected. This raw material, strongly recalling the red flint from the Ludian stage of the Eocene epoch, has already been found in several other regional occupations (blades or other tools). Among the artefacts preserved in situ, we identified several other burins and, to a lesser extent, scrapers and piercers. A few backed bladelets were mixed with the debitage products close to the northwest edge of the hearth.

A reindeer antler point was also removed from the rest of the archaeological floor before the excavated surface was refilled. This antler point was discovered less than 2 metres from the west edge of the hearth and a few centimetres away from a large worked reindeer antler fragment. It has a subrectangular section and presents a double bevel as well as a longitudinal groove. The morphology of this point is very similar to other examples known in the regional Magdalenian.

All the rocks have been left in situ, and the information concerning this category of artefact is only based on field observations. They are characterized by their morphological and petrographical diversity, and include mainly sandstone, sandstone-shale and limestone.

Apart from rocks contained in the hearth, probably reserved for its functioning, some other large blocks could have composed elements of the infrastructure in view of their size and weight. Such elements are known in the other Magdalenian occupations of the Paris Basin where they sometimes delimited a circle several metres in diameter. One of the main interests of this occupation lies in the presence of numerous bone remains. Almost all the determined elements relate to the reindeer. The discovery of reindeer tarsi in anatomical connection and the presence of skeleton elements particularly sensitive to taphonomical depletion, like antler fragments, ribs and vertebrae, obviously show exceptional preservation conditions and the absence of post-depositional disturbances. A first estimation based on teeth series and forelimbs (the radius fragments), gives a Minimum Number of Individuals of two reindeers brought back to the occupation site. At present, the absence of teeth series belonging to very young individuals prevents a more accurate seasonal determination of the Magdalenian visits. However, the presence of a large-sized antler from a hunted animal, belonging to an adult male reindeer, permitted the processing of this specimen to be 
estimated at between September and mid-December. The occupation would thus have taken place in the autumn or at the beginning of the winter, unless it is considered that the reindeer antler could have been transported for several months as a stock of raw material. Besides the reindeer remains, we observed a diaphysis fragment with dimensions suggesting an attribution to a horse or a bovine, and the horse skull discovered outside the occupation site.

All the artefacts are located around the pit hearth, close to a large ochrestained area. The low density of remains suggests a short-term residence. A small pit was identified close to the hearth. Considering the bone remains, we note an asymmetrical distribution in relation to the hearth. There are a lot of bone fragments to the south of the fire area, while they are almost absent to the north. Such a pattern probably points to the repetitive disposal behaviour of the prehistoric people. Two debitage product concentrations, related to bladelet production, are located to the north-east of the hearth. Two other flint concentrations seem to be more probably toss zones.

Finally, we observe a group of tertiary flint blades near a large sandstone slab. This group could be interpreted as a spare space of unused blades. With regard to the spatial organization, some levels of Pincevent (Seine-etMarne) offer the most striking parallels.

Two bone fragments were sent to a laboratory in order to obtain radiocarbon dates. The first one, a reindeer radius fragment which was collected close to the hearth, delivered a date of 12,000 \pm 70 BP. The second one, a cheek tooth from the isolated horse skull, delivered a date of 12,230 \pm 70 BP. These results are very similar to those obtained for the nine other Paris Basin Magdalenian sites. We hope that OSL (Optically Stimulated Luminescence) analysis (in progress) could provide this Upper Magdalenian occupation with an accurate chronological context through the Weichselian Late Glacial chronozones.

The outstanding conservation of this occupation site already makes La Haye one of the most important site for regional Magdalenian studies. The analysis of this occupation will be completed by exhaustive excavations. Since the planned building project has been cancelled, the site area is no longer threatened. However, new excavations are scheduled for the coming years.

Keywords

Upper Magdalenian, Paris Basin, chronology, geoarchaeology, spatial organization.

\section{INTRODUCTION}

Suite à un projet d'implantation d'un circuit automobile, des investigations archéologiques ont été réalisées au cœur du territoire Seine-Aval entre mai et septembre 2009. La surface explorée, située sur la rive gauche et en bordure immédiate de la Seine, couvre plus de 100 hectares. Elle s'étend sur deux communes : Flins-sur-Seine et les Mureaux (fig. 1). De nombreux ensembles archéologiques diachroniques y ont été repérés. Ils indiquent que le secteur a été plus ou moins intensément occupé depuis le début du Paléolithique jusqu'à l'Antiquité tardive (Debout dir., 2009). Les aspects géomorphologiques nous ont conduits à distinguer une partie nord et une partie sud (fig. 1) : la partie nord, située en bordure du lit mineur du fleuve, est caractérisée par des ensembles sédimentaires d'origine alluviale; la partie sud est essentiellement constituée de dépôts sédimentaires plus anciens (terrasse saalienne) sur lesquels reposent des unités sableuses colluviales d'âge holocène.

Globalement, la partie nord présente le contexte le plus favorable à la conservation des vestiges, toutes périodes chronologiques confondues. On y recense de nombreuses structures néolithiques, protohistoriques et gallo-romaines. C'est aussi à cet endroit qu'un niveau d'habitat, bien conservé, rapporté au Magdalénien supérieur a été découvert. Les vestiges de cette occupation se distribuent sur le territoire des deux communes mentionnées plus haut (fig. 1). La concentration principale, dénommée «zone est», est localisée sur le lieu-dit de la Haye, aux Mureaux, tandis que les vestiges mis au jour à 500 mètres en direction de l'ouest, dans une position stratigraphique analogue, se situent sur la commune de Flins-sur-Seine. Cette dernière zone présente par ailleurs un grand intérêt puisqu'elle livre une succession stratigraphique qui semble couvrir toute la période du Tardiglaciaire weichsélien. 


\section{CONTEXTE ET PREMIER APERÇU DES DECOUVERTES}

\section{La zone est : une unité d'occupation magdalénienne remarquablement conservée}

Dans la zone est, le niveau d'habitat, riche en vestiges lithiques et osseux, est apparu à une profondeur de $1,50 \mathrm{~m}$ (soit à 21,40 m NGF). Dès le dégagement des premiers mètres carrés du sondage élargi, nous avons constaté que les objets reposaient sur un sol relativement plan et qu'ils matérialisaient une unité d'occupation structurée. La présence d'un foyer aménagé au moyen de blocs rocheux, de nombreux restes osseux et d'une nappe d'ocre rouge témoignait d'un état de conservation remarquable (fig. 2). Ce sont avant tout les caractéristiques typotechnologiques de l'industrie lithique qui ont permis une première évaluation de l'attribution culturelle de cet ensemble. Les débitages laminaires réalisés au percuteur tendre organique, la présence de grattoirs sur lame, de burins et de lamelles à dos nous ont rapidement incités à évoquer un rapprochement avec les industries magdaléniennes d'Île-de-France (Audouze et al., 1988; Valentin et Pigeot, 2000). Un premier examen des témoins osseux, révélant la présence du renne, a conforté cette attribution. Enfin, le contexte stratigraphique offrait lui aussi quelques analogies avec celui d'autres sites magdaléniens du Bassin parisien. En effet, la couche d'alluvions renfermant le niveau d'habitat, composée de sable et de limon argileux, et épaisse de plus de 2 mètres en moyenne, s'apparente aux dépôts d'inondation au sein desquels les vestiges magdaléniens ont été ensevelis dans d'autres gisements de la vallée de la Seine, notamment à Pincevent et à Étiolles (Roblin-Jouve, 1994). Ainsi, nous avons rapidement disposé d'un faisceau d'indices pour inscrire cette occupation paléolithique mise au jour sur le lieu-dit de la Haye au sein du Magdalénien et plus précisément au cours de sa phase supérieure. Cette attribution a été confirmée, quelque temps après, au moyen de deux datations radiocarbone (voir ci-dessous).

Les conditions d'ensevelissement du niveau d'occupation de la Haye ont été telles que les vestiges se sont trouvés immobilisés sans subir de déplacements postdépositionnels notables. De toute évidence, cette zone

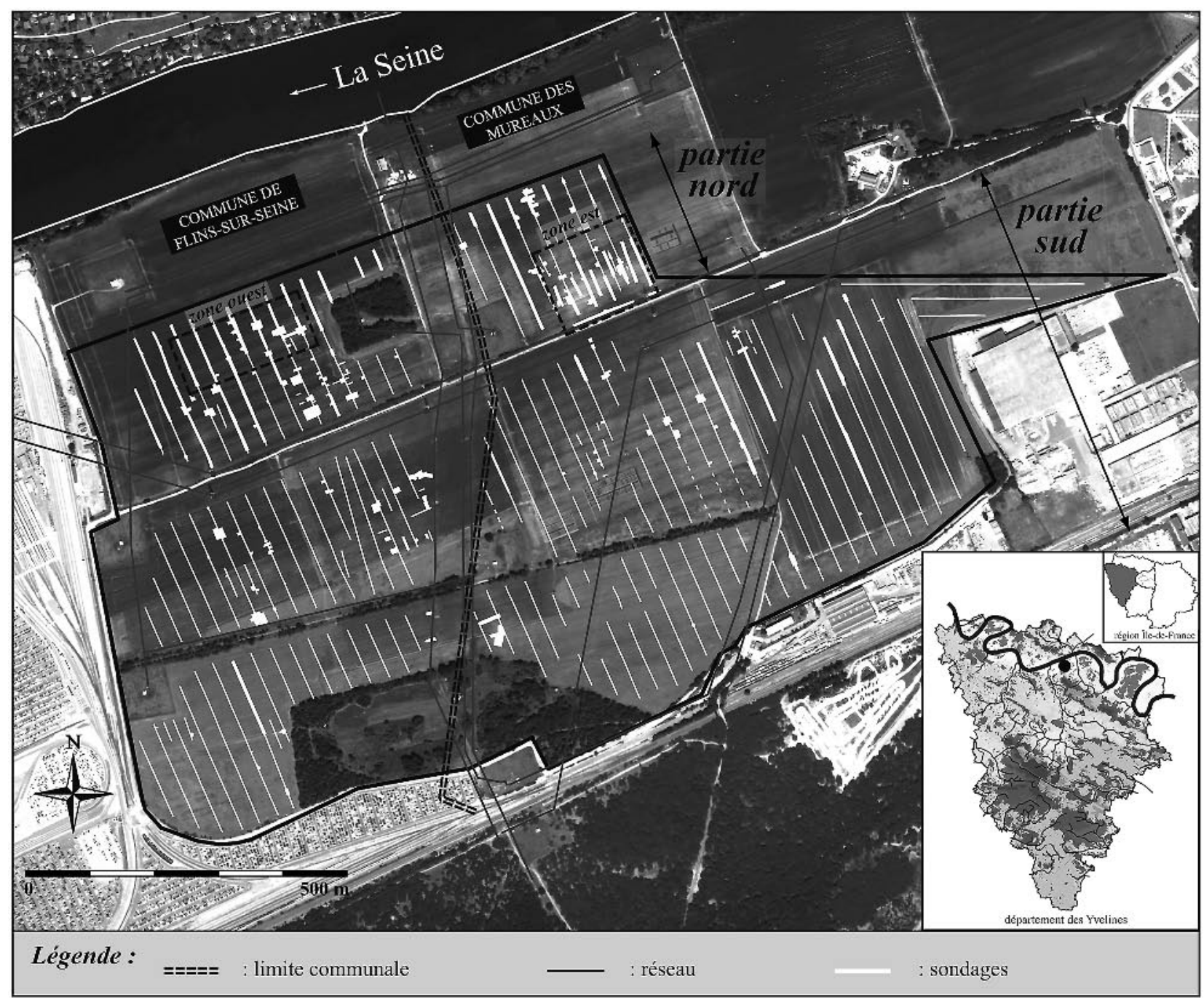

Fig. 1 - Plan général de l'emprise sondée sur les communes de Flins-sur-Seine et des Mureaux (Yvelines).

Fig. 1 - General map of test pits at Flins-sur-Seine and Les Mureaux (Yvelines). 
conservée de manière remarquable exigeait une étude minutieuse qui ne pouvait être envisagée dans le cadre strict d'une opération d'archéologie préventive. Aussi, en accord avec une commission composée de représentants du SRA et de la CIRA, la décision a été prise de laisser en place les vestiges dégagés dans le sondage élargi pour une fouille ultérieure et de reboucher la zone excavée afin de la protéger. Ce choix s'est révélé d'autant plus raisonnable que le projet de construction du circuit automobile est actuellement abandonné et

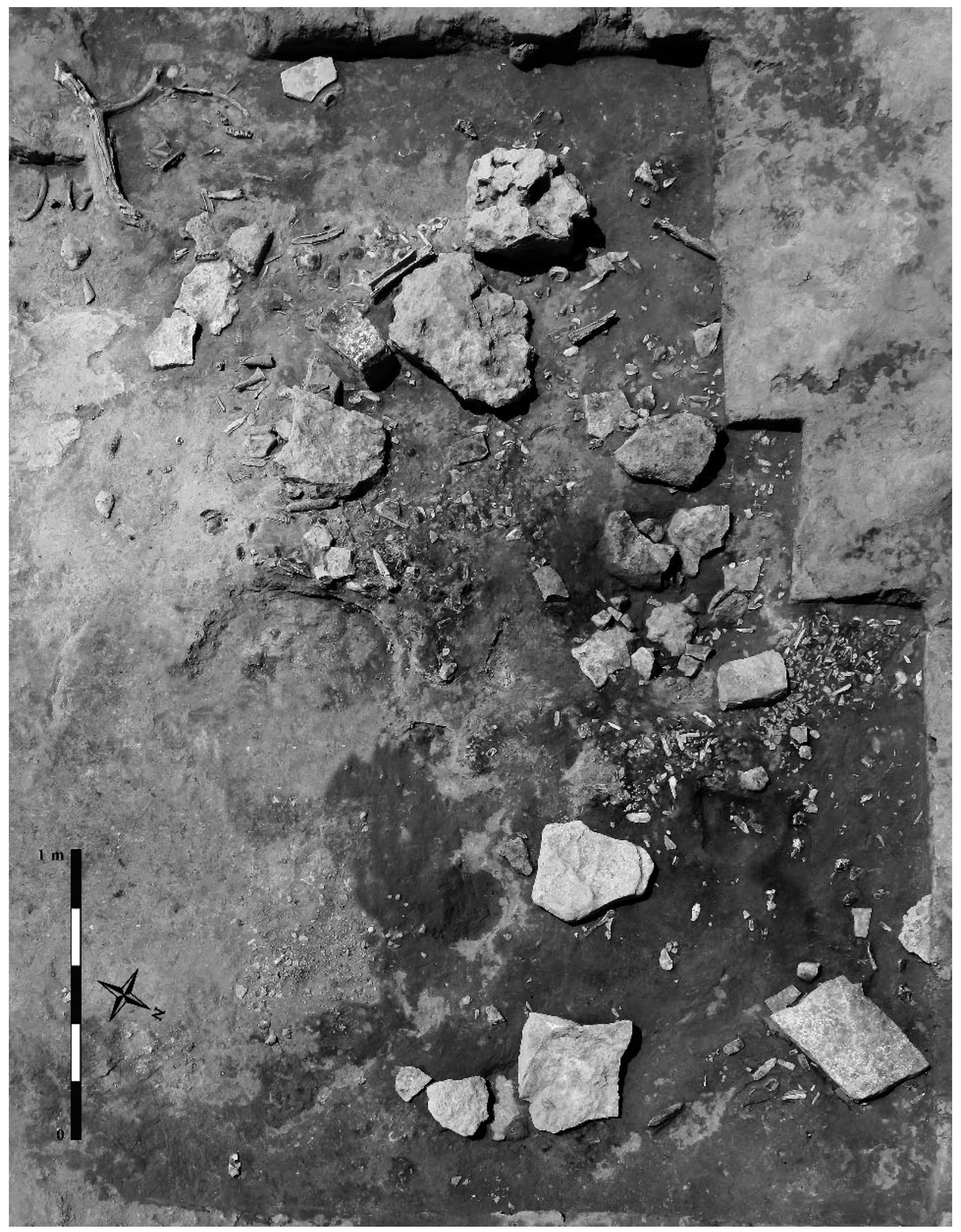

Fig. 2 - Vue verticale de la partie fouillée du niveau d'occupation magdalénien de la Haye (cliché P. Laforest).

Fig. 2 - Vertical view of the Magdalenian horizon occupation unit at La Haye (photo P. Laforest). 
que la menace pesant sur ce site est temporairement levée. Les travaux de terrain ont donc principalement consisté à recueillir des informations essentielles permettant de caractériser la nature de cette occupation et de déterminer son extension. Seuls les objets les plus fragiles, qui auraient été détériorés par les opérations de rebouchage, ont été prélevés.

Les tranchées et sondages réalisés en périphérie de cette concentration et dans les zones au sein desquelles les observations géomorphologiques indiquaient l'existence de la couche d'alluvions ont permis de circonscrire l'étendue de l'occupation. On peut ainsi estimer à 200 mètres carrés la surface riche en vestiges. Par ailleurs, un crâne de cheval isolé découvert à une centaine de mètres en direction de l'ouest (fig. 3) pourrait représenter un rejet éloigné appartenant à la même occupation (voir ci-dessous).

\section{La zone ouest : une structure en pierre et une séquence tardiglaciaire développée}

La zone ouest, dont les découvertes restent moins importantes que celles de la zone est, présente surtout un intérêt pour la séquence naturelle du Tardiglaciaire qui a pu y être documentée. Les descriptions stratigraphiques seront exposées un peu plus loin, mais il faut d'ores et déjà mentionner la mise au jour d'une structure pierreuse (fig. 4), dont la fonction est actuellement indéterminée, à la base de la séquence. Cette concentration de blocs rocheux a été dégagée au sommet d'une couche limono-sableuse qui trouve de bonnes correspondances avec celle qui contient l'occupation magdalénienne de la zone est. Contrairement au riche niveau d'habitat laissé en place, elle a été entièrement démontée; les éléments particuliers qu'elle contenait (voir ci-dessous) suggèrent l'existence d'une relation entre les deux zones, même s'il reste impossible d'établir leur stricte contemporanéité.

\section{LES ASPECTS GÉOARCHÉOLOGIQUES ET CHRONOSTRATIGRAPHIQUES DU GISEMENT}

D'après les cartes géologiques du BRGM (feuilles de Pontoise et de Versailles), les zones de découvertes archéologiques sont localisées à la jonction d'un complexe de terrasses anciennes (Fy, dit «terrasse de la cote $25 »)$ et d'alluvions récentes $(\mathrm{Fz})$. Au nord, la Seine entre directement en contact avec les formations du Crétacé (C5 et C6) surmontées par les dépôts tertiaires que l'on rencontre habituellement dans le Bassin parisien (fig. 5). Le gisement est situé légèrement en aval de la partie la plus concave d'un méandre du cours d'eau qui confère un aspect fortement dissymétrique au fond de vallée. Le contexte topographique est donc particulièrement favorable à une sédimentation alluviale. La topographie du terrain est marquée par la présence d'un talweg central à sec recoupant les diverses terrasses et séparant le terrain sondé en deux espaces présentant une conservation différente des dépôts tardiglaciaires (fig. 6).

\section{La zone est}

Deux grandes coupes ont été réalisées de part et d'autre de la zone d'occupation magdalénienne afin de documenter les aspects stratigraphiques. Nous présentons ici celle qui est située immédiatement à l'est (fig. 7). Les vestiges magdaléniens sont interstratifiés dans une épaisse couche d'alluvions qui est venue combler un chenal hérité d'une paléotopographie pléniglaciaire. Ce dernier est encadré au nord par le versant d'une ride de grave et au sud par la terrasse ancienne. Cette position géographique a fortement conditionné la dynamique de dépôt qui n'a cessé de déplacer le cours d'eau en direction du sud. Le contexte sédimentaire, au sein duquel on note l'absence de phénomènes érosifs, est particulièrement propice à la conservation en place des vestiges archéologiques. Si le chenal avait été géographiquement plus stable, la dynamique de dépôt aurait été moins prononcée, et une divagation du chenal vers le nord aurait sans doute érodé le site.

Il est impossible de déterminer si ce chenal était en eau durant le séjour des Magdaléniens. Les lits limonosableux à vergence sud qui composent la partie inférieure de la séquence de comblement sont d'une remarquable continuité et indiquent un remplissage graduel et continu. Cette couche litée d'alluvions a ensuite été recouverte d'une unité sableuse plus homogène qui a isolé le niveau archéologique de l'influence de la pédogénèse et de l'érosion, sauf, peut-être, dans la partie nord.

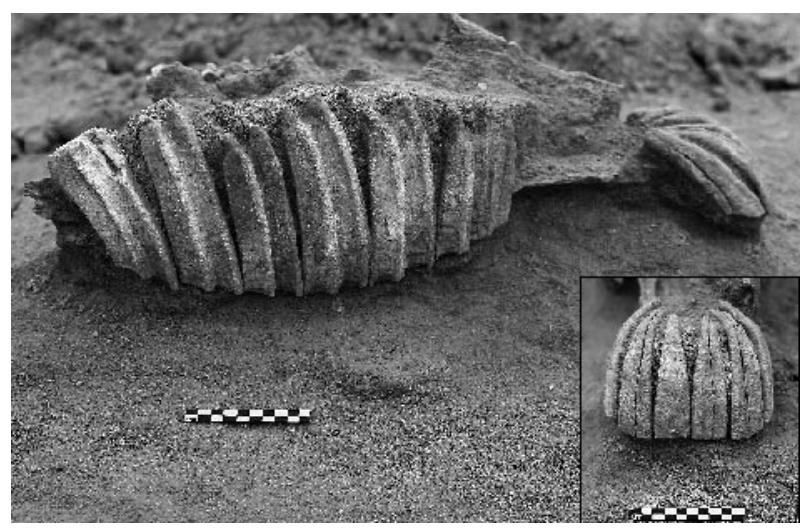

Fig. 3 - Crâne de cheval découvert en périphérie du centre de l'occupation. Fig. 3 - Horse cranium found on the margin of the occupation centre.

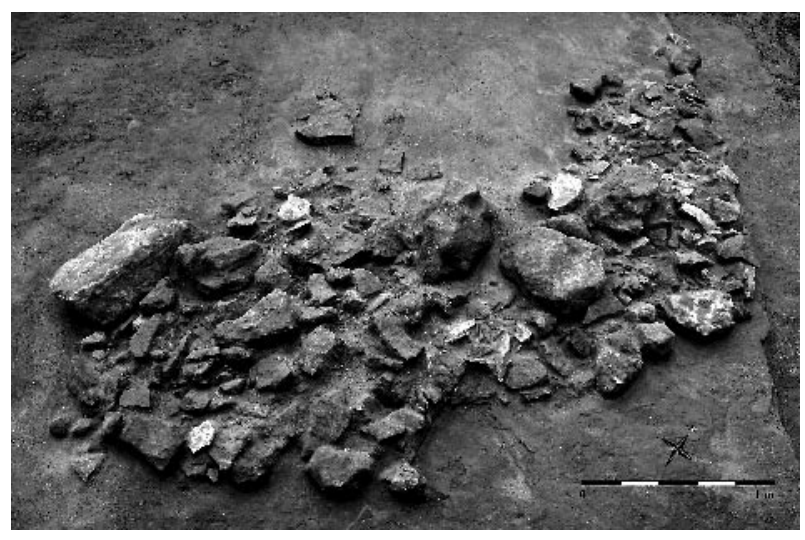

Fig. 4 - Structure pierreuse dégagée dans la zone ouest. Fig. 4 - Stone structure found in the western zone. 
De l'autre côté de la ride de grave, on retrouve, appuyés directement sur le versant, des sédiments essentiellement limono-argileux. Il s'agit de «limons de débordements » que l'on rencontre habituellement au sommet des séquences alluviales holocènes dans les vallées du Bassin parisien (Pastre et al., 1997 et 2002). La présence d'un chenal holocène très érosif n'y a pas permis la conservation d'éventuels dépôts tardiglaciaires. Cependant, et comme l'indique la topographie actuelle, la séquence tardiglaciaire semble s'étendre en direction de l'est. En effet, lors de fouilles réalisées aux Mureaux par Y. Barat en 1988 (com. orale et documents non publiés), une séquence identique à celle contenant les vestiges de la zone est a été observée.

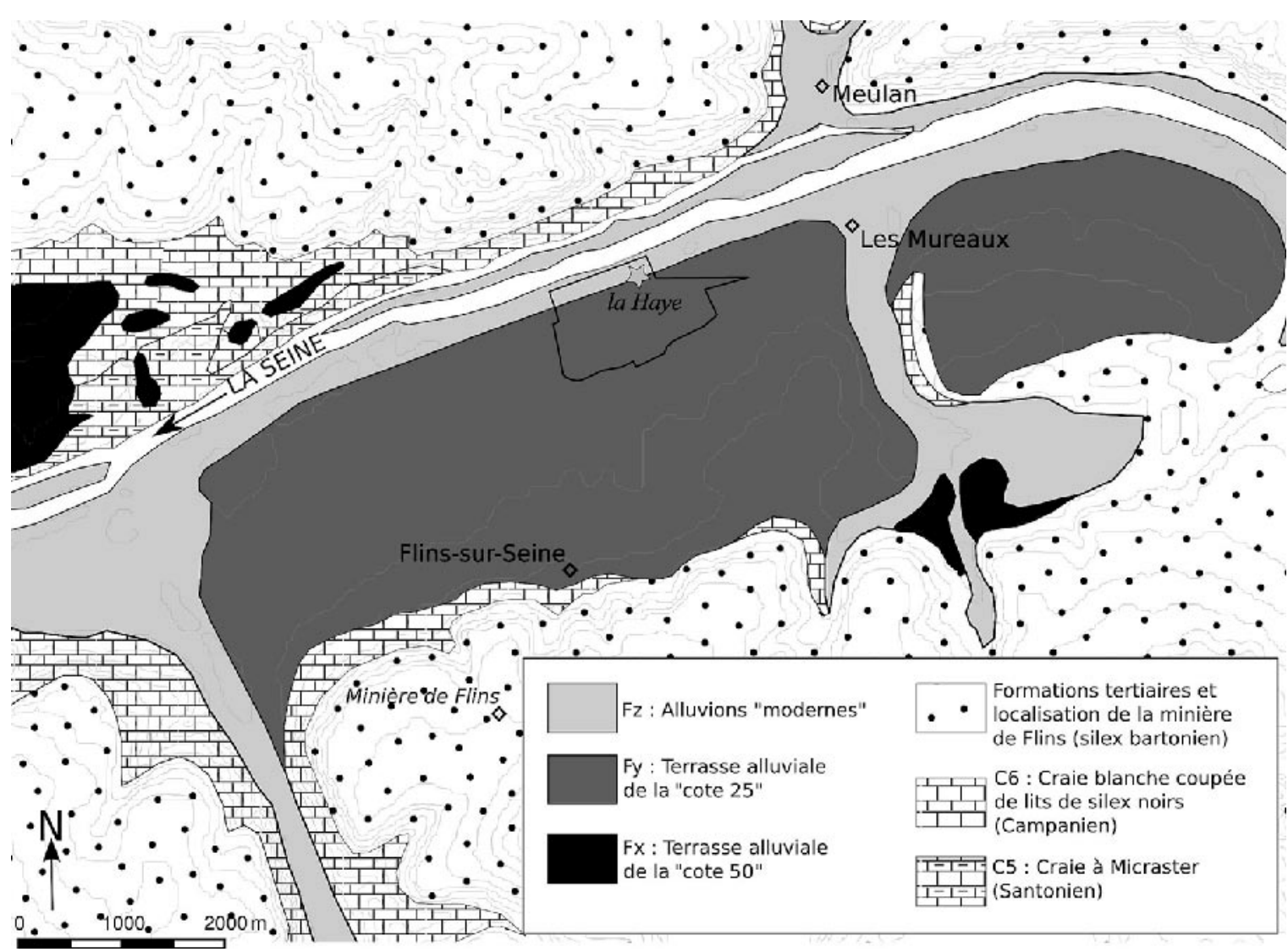

Fig. 5 - Contexte géologique du gisement de la Haye (carte simplifiée d'après les données du BRGM, feuilles Pontoise et Versailles). Fig. 5 - Geological context of La Haye (simplified map after BRGM data, Pontoise et Versailles sheets).

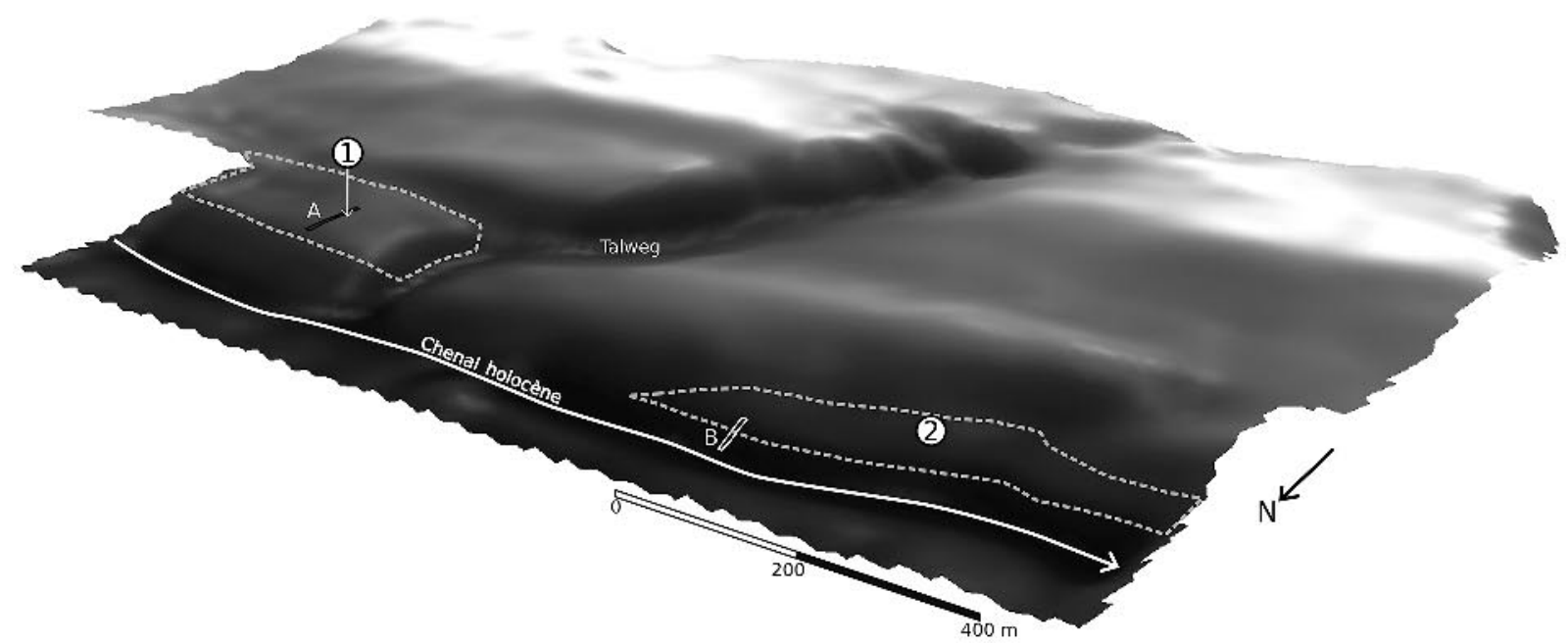

Fig. 6 - Vue en 3 dimensions de l'environnement topographique de la Haye (représentée avec une exagération de x 10). Trait pointillé : position des séquences tardiglaciaires; 1 : l'occupation magdalénienne de la zone est; 2 : la structure pierreuse de la zone ouest; A : coupe stratigraphique présentée figure 7 ; B : coupe stratigraphique présentée figure 8.

Fig. 6 - Three-dimensional view of the topographical environment of La Haye (amplified x 10). Dotted line: position of Late Glacial layers 1: Magdalenian horizon occupation unit of the eastern zone; 2: Stone structure in the western zone; A: Stratigraphic section presented in figure 7; B: Stratigraphic section presented in figure 8. 
SUD
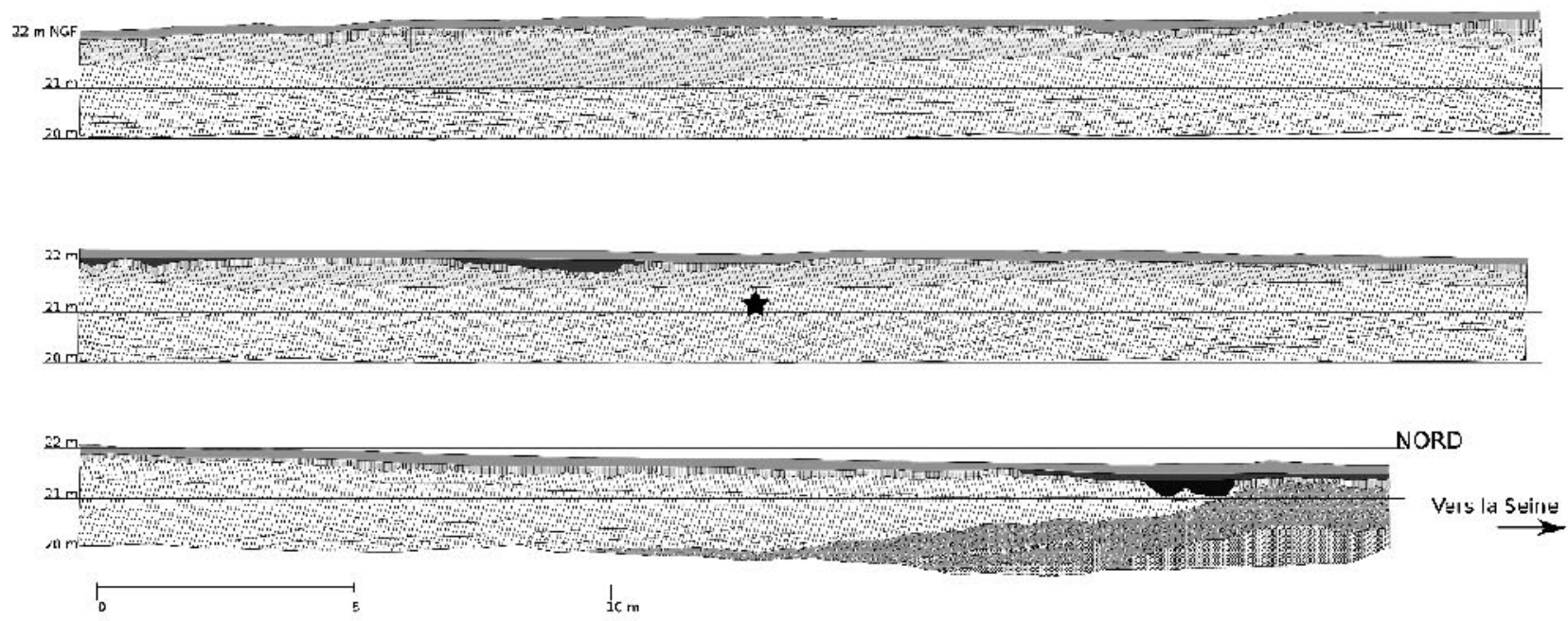

Sol ectuel chargé en "boues jarisiennes"

Liman sasle. $\mathrm{x}$ bruın à gris-br.un

(colluxions sableuses pédogénéisées, niveaux féolithiques!

|||||| | Limon sableux très oxydès (horizon Bt holocène;

Limon b-un (remplissage s'une structure arc félogique)

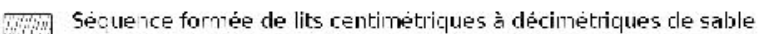

Limon sab|cux à poresité marq Jêc, préscnce de Ientilles de cərbonates

Fig. 7 - Détail de la coupe A réalisée à proximité de l'occupation magdalénienne de la zone est.

Fig. 7 - Detail of the cross-section A, in the immediate vicinity of the Magdalenian occupation in the eastern zone.

Ces dépôts étaient également constitués de lits limonosableux et présentaient aussi un net pendage vers le sud. Il est donc possible de considérer l'ensemble des terrains situés entre le centre de l'agglomération des Mureaux et la Haye, dont les altitudes s'échelonnent entre 21,5 et 23 mètres NGF, comme les témoins résiduels d'une terrasse tardiglaciaire susceptible de contenir des niveaux magdaléniens bien conservés.

\section{La zone ouest}

La zone ouest est localisée sur le versant nord d'une ride de terrasse ancienne parallèle au cours actuel de la Seine (fig. 6). Les travaux de terrain ont mis en évidence la présence de traces importantes d'oxydation se développant au-dessus des niveaux de graves et de sables. Elles étaient parfois recouvertes d'une unité sableuse brune épaisse de quelques dizaines de centimètres. Il reste difficile de proposer une datation pour cette unité alluviale ancienne. L'importance des traces d'oxydation et l'écart d'altitude avec le sommet de la séquence tardiglaciaire de la zone est (au moins 2 mètres de plus) permettent toutefois d'exclure l'hypothèse d'une attribution de ces dépôts détritiques à la glaciation du Weichsélien. Une comparaison avec les terrasses anciennes connues dans le nord-ouest de la France (Antoine et al., 2007) suggère un rapprochement avec le complexe Saalien pour les dépôts de grave sableuse et avec la pédogenèse éémienne pour les traces d'oxydation.
La séquence tardiglaciaire rencontrée dans cette zone ouest est relativement bien conservée. La paléotopographie particulière, liée à la mise en place d'un cône alluvial déjeté associé au talweg central pendant le Saalien, a protégé en partie ces dépôts de l'érosion alluviale holocène. La séquence apparaît plus dilatée dans le temps que celle de la zone est. Elle est en outre plus complexe et regroupe davantage de faciès sédimentaires (fig. 8). Pour certains de ces derniers, il est possible de proposer une attribution chronologique en comparant les faciès sédimentaires relevés à la Haye avec ceux décrits pour d'autres séquences tardiglaciaires du Bassin parisien (Pastre et al., 2002 et 2003) :

- les faciès terminant la séquence peuvent être associés au Dryas récent. Ces limons calcareux, parfois sableux, sont caractéristiques et communément marqués par la pédogénèse du début de l'Holocène;

- le faciès situé immédiatement sous ces dépôts correspond à un «sol gris », parfois très riche en charbons de bois, que l'on peut rattacher à l'Allerød ;

- les niveaux antérieurs correspondent à la couche limono-sableuse litée au sein de laquelle a été découverte l'occupation magdalénienne de la zone est. Il faut toutefois préciser que ces faciès sont ici moins épais, d'aspect légèrement plus sableux et qu'ils ne présentent pas de grandes unités limoneuses. Pour le moment, ils ne peuvent être rapportés à une chronozone précise. Cependant, la similitude entre ces dépôts limono-sableux lités et ceux rencontrés à Pincevent et à Étiolles (Roblin-Jouve, 1994; Orliac, 2006; Chabrol 
et al., 2008) suggère attribution au Dryas ancien ou au Bølling. Le Dryas moyen semble pouvoir être exclu en raison de la puissance des dépôts observés ici.

Le niveau rapporté à l'Allerød a été tronqué lors du dépôt des limons du Dryas récent. Aussi, la conservation de vestiges archéologiques dans cette unité stratigraphique ne peut être envisagée que sur une bande relativement étroite et parallèle au cours du fleuve. Cela étant, de nombreuses zones de combustion d'origine naturelle y ont été repérées sous la forme de concentrations de charbons et de témoignages d'altération thermique du sol (rubéfaction). Aux endroits où le sol allerød était érodé, ces aires de combustion apparaissent au sommet de l'unité de grave sableuse sous-jacente. On peut rappeler que les incendies tardiglaciaires sont étroitement liés aux variations climatiques et à la nature du couvert végétal, et qu'ils se propagent plutôt pendant les périodes sèches des phases de déglaciation (Marlon et al., 2009). La fin de l'Allerød (post-IACP) et le début du Dryas récent sont des périodes favorables au développement de ce type de phénomène, surtout en raison du fort développement des forêts de pins. On en retrouve le témoignage dans plusieurs gisements d'Europe de l'Ouest, notamment sur celui du Closeau (Rueil-Malmaison, Hauts-deSeine; Bodu dir., 1998), distant des Mureaux d'une vingtaine de kilomètres. Si l'on se réfère aux déterminations des charbons de bois de ce gisement (voir notamment Pernaud in Bodu dir., 1998; Pastre et al., 2000), Pinus sylvestris devrait également être l'espèce dominante, voire exclusive, dans les zones de combustion observées à la Haye. Des datations radiocarbone et des analyses anthracologiques devront être effectuées pour vérifier cette hypothèse et, le cas échéant, permettre d'établir un calage chronologique précis de ce phénomène naturel dans cette partie de la vallée de la Seine.
Les faciès sédimentaires attribués au Dryas récent sont fortement érodés, mais peuvent présenter localement un potentiel archéologique; ce dernier reste tout de même faible pour le faciès sableux inférieur qui semble lié à des dépôts de très haute énergie. En ce qui concerne la séquence alluviale holocène, la sédimentation est essentiellement détritique avec une succession de couches limoneuses plus ou moins argileuses et/ou marquées par une présence plus importante de matière organique. Il s'agit là d'une séquence classique de limons argileux qu'il faut probablement situer pendant le Subatlantique ou à la fin du Subboréal (Pastre et al., 1997 et 2002; Le Jeune et al., 2005). Un sol de rive recoupe les dépôts tardiglaciaires antérieurs et correspond à la variation latérale des unités colluviales associées à une occupation néolithique en place. Il est antérieur aux dépôts limoneux, lesquels sont principalement postérieurs à l'âge du Bronze dans le Bassin parisien. Il faut donc considérer ce niveau comme un sol de rive du début de l'Holocène, sans qu'il soit possible de préciser davantage sa datation.

\section{Synthèse stratigraphique}

Les observations stratigraphiques réalisées lors des travaux de terrain ont permis de mettre en évidence deux contextes particuliers donnant lieu à la conservation de séquences tardiglaciaires (fig. 6 et 9). La zone est, comprenant l'occupation magdalénienne, présente une conservation liée à un héritage morphogénétique probablement pléniglaciaire. Un ancien chenal est remis en eau au cours du Tardiglaciaire lors d'épisodes de crue, apportant des sédiments de manière rythmée (litage). Cette séquence détritique est particulièrement favorable à la conservation in situ de vestiges. La ride de grave a protégé ces dépôts de l'érosion alluviale holocène.

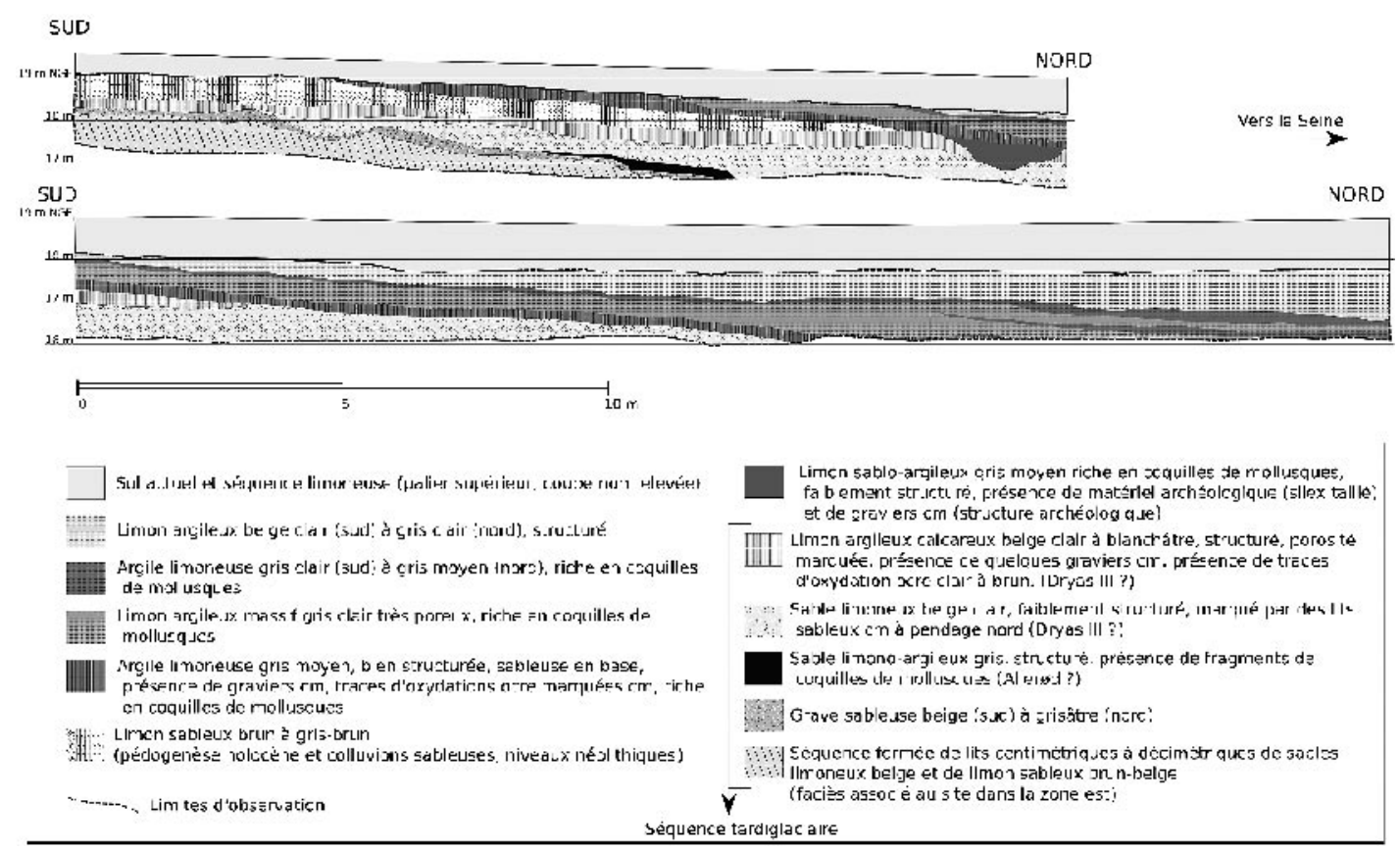

Fig. 8 - Détail de la coupe B illustrant la séquence tardiglaciaire de la zone ouest.

Fig. 8 - Detail of the cross-section B showing the Late Glacial layers of the western zone. 
La zone ouest montre, pour sa part, une conservation de rive liée à la présence d'un obstacle en amont. Ce sont les dépôts antérieurs de grave, formant un relief immédiatement à l'ouest de l'embouchure du talweg (relique d'un cône alluvial déjeté), qui ont protégé la rive tardiglaciaire de l'érosion liée au fonctionnement du chenal holocène. Ce contexte permet d'observer une succession stratigraphique comprenant des faciès sédimentaires limoneux lités (renfermant les occupations magdaléniennes sur les sites de la Haye, de Pincevent et d'Étiolles), un «sol gris» postérieur attribué à l'Allerød et les limons calcareux du Dryas récent, identifiés à plusieurs reprises en d'autres endroits du Bassin parisien (fig. 8).

La question du calage chronologique des séquences limoneuses litées contenant les occupations magdaléniennes en Île-de-France demeure ouverte, et les observations réalisées sur le site de la Haye permettent d'en débattre à nouveau. En effet, si les datations radiométriques obtenues sur les sites d'Étiolles et de Pincevent semblent indiquer une possible attribution des occupations magdaléniennes au Bølling (voir cidessous), les faciès sédimentaires très détritiques, à l'instar de ceux de la Haye, paraissent incompatibles avec cet interstade qui, dans la région, a été pour l'instant identifié sous la forme de dépôts essentiellement organiques (Pastre et al., 2000). Pour tenter de démêler cet écheveau, nous avons réalisé plusieurs prélèvements à la Haye en vue de datations OSL. Ces dernières permettront peut-être de préciser la chronologie de la séquence limoneuse litée qui contient l'occupation magdalénienne et celle des ensembles sédimentaires qui l'encadrent (fig. 9). Soulignons que la remarquable constance des dépôts suggère une mise en place au cours d'une phase climatique plutôt stable. Les analyses en cours visent, pour l'heure, à obtenir plusieurs dates à partir d'échantillons provenant de la base de cette séquence litée et du sommet de la grave sousjacente. Gageons qu'elles révéleront le terminus post quem à partir duquel le dépôt des lits limono-sableux contenant l'occupation s'est amorcé.

\section{Les dates radiocarbone}

Dans l'attente des résultats des analyses OSL, qui permettront peut-être de proposer un calage précis de l'ensemble sédimentaire contenant les vestiges magdaléniens, deux échantillons ont été envoyés en laboratoire afin d'obtenir des datations radiocarbone. Le premier, un fragment de radius de renne recueilli à proximité du foyer, a livré la date de $12000 \pm 70 \mathrm{BP}$ (Beta-265097). Le second, une dent provenant du crâne de cheval isolé découvert à une centaine de mètres à l'ouest de la concentration principale, a livré une date de $12230 \pm 70$ BP (Beta-265098). Par ailleurs, un âge de $12770 \pm 120 \mathrm{BP}\left(\right.$ date $\left.{ }^{14} \mathrm{C}\right)$ a récemment été mesuré sur une esquille d'os indéterminé provenant de l'occupation magdalénienne de l'abri-sous-roche de la CôteMasset à Bonnières-sur-Seine. Ce sont ainsi les deux plus importants gisements des Yvelines qui bénéficient aujourd'hui de calages chronologiques. Nous rappellerons que sur la quarantaine de gisements recensés dans le Bassin parisien, huit seulement, dont cinq en Île-de-France, avaient jusqu'alors fait l'objet de tentatives de datation. Désormais, ce sont donc 10 gisements magdaléniens qui disposent d'au moins une date radiocarbone (tabl. 1).

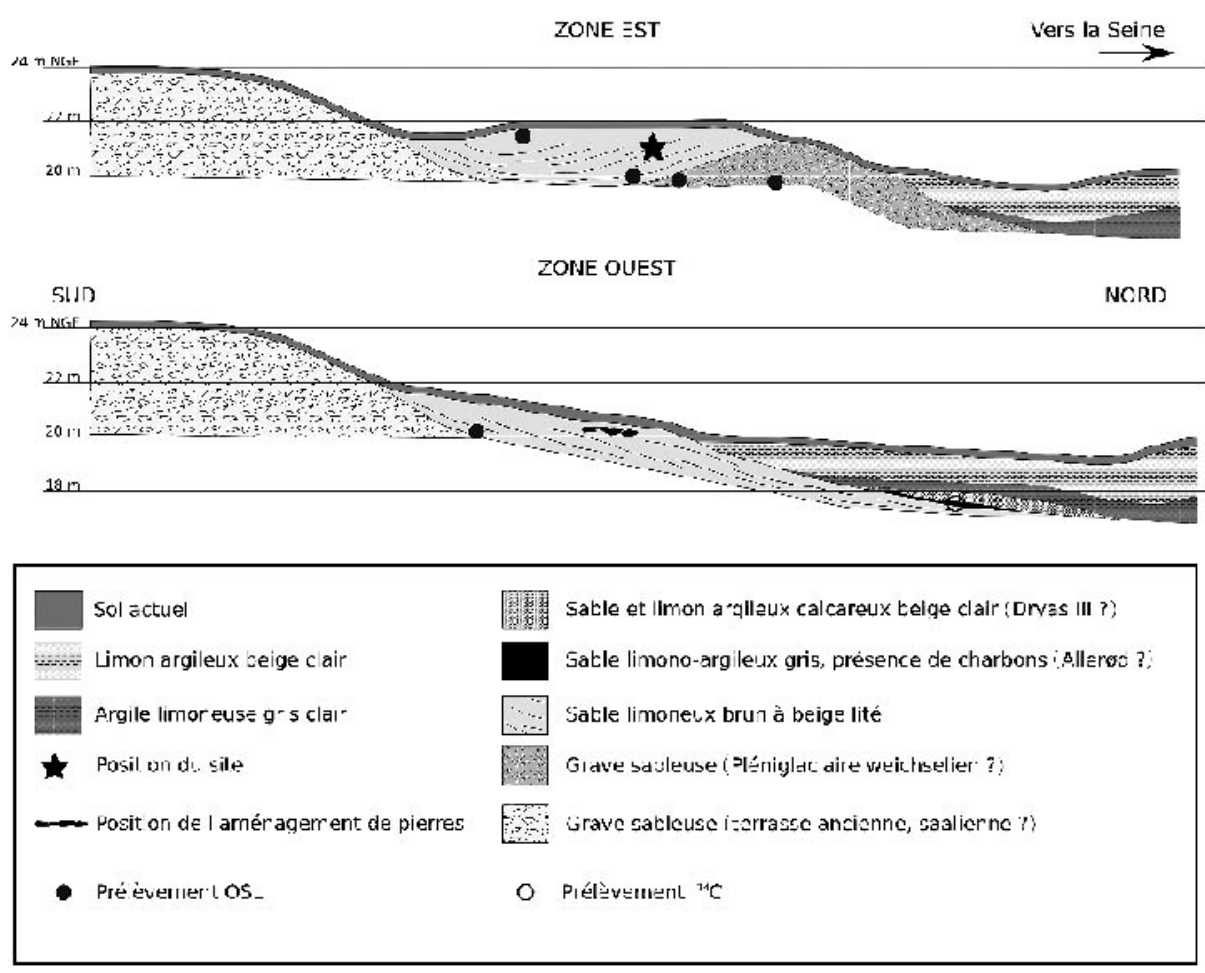

Fig. 9 - Synthèse stratigraphique schématique des zones ouest et est.

Fig. 9 - Stratigraphical synthesis of the eastern and western zones. 
D'après les pics de probabilités qui se dégagent des courbes, l'abri-sous-roche de la Côte-Masset livrerait l'occupation la plus ancienne tandis que celle de la Haye figurerait plutôt, comme Pincevent, parmi les plus récentes (fig. 10). Cependant, c'est avec beaucoup de prudence qu'il faut considérer la succession chronologique suggérée par le graphique proposé ici compte tenu de l'imprécision de la méthode radiocarbone et de la présence d'un plateau d'âges constants sur la courbe de calibration. De plus, il n'est pas totalement pertinent de comparer des courbes obtenues à partir d'une seule date avec d'autres courbes qui en intègrent jusqu'à 12 . En revanche, les dates dont on dispose actuellement sont suffisamment nombreuses pour fournir une première estimation de la position chronologique globale du Magdalénien supérieur de la région. Les pics de probabilités les plus élevés s'inscrivent aujourd'hui dans un intervalle couvrant plus d'un millénaire et dont les bornes se situent à 13300 et 11950 BC. C'est aussi durant cet intervalle que, semble-t-il, les groupes creswelliens et hambourgiens, dont on peut parfois mettre en évidence l'influence dans certaines industries magdaléniennes du Bassin parisien, s'établissent en Angleterre et dans le nord de l'Allemagne (Jacobi, 1991; Grimm et Weber, 2008; Valentin, 2008).
L'étendue de l'intervalle ne permet néanmoins pas de déterminer s'il faut caler le Magdalénien supérieur du Bassin parisien à la toute fin du Dryas I, au cours du Bølling ou du Dryas II. L'hypothèse d'une diffusion au cours des trois chronozones n'est pas à exclure malgré l'unité socio-économique que révèlent depuis longtemps les analyses de matériel des divers sites concernés. Dès lors, on saisit mieux l'enjeu des possibles corrélations géologiques, au moyen d'analyses OSL notamment, que l'on tente de mener aujourd'hui à la Haye et qu'il conviendrait de poursuivre sur les gisements de Pincevent et d'Étiolles, actuellement en cours de fouille. C'est aussi à partir des analyses paléoenvironnementales que l'on devra tenter d'obtenir des informations complémentaires. Certes, les pollens font défaut dans ce type de séquence détritique (Leroyer, 1994), mais les résultats récemment obtenus par l'analyse des charbons sur quelques sites du Bassin parisien, piste qui avait été abandonnée depuis plus d'une dizaine d'années, font naître de nouveaux espoirs (Bodu et al., 2008 et 2009). Compte tenu du bon état de conservation du niveau d'occupation de la Haye, les charbons devraient être conservés en grand nombre. Ils permettront vraisemblablement d'établir quelques corrélations avec les

\begin{tabular}{|c|c|c|c|c|c|c|}
\hline Sites & Iocal, strati. & Néthode & Echantillon & Réf. lab. & Résultats BP & Sigma \\
\hline \multirow{2}{*}{$\begin{array}{l}\text { Le Prí-des-Forges } \\
\text { (Marsaugy; Yonne) }\end{array}$} & unitć Dl4 & $\Lambda \mathrm{MS}$ & Os & $0 \times A 740$ & 12120 & 200 \\
\hline & unité N19 & AMS & $O s$ & $0 \times A$ & 12140 & 75 \\
\hline \multirow{2}{*}{$\begin{array}{c}\text { La Ilave } \\
\text { (Les Mureaux ; Yvelines) }\end{array}$} & unité G13 & AMS & $\mathrm{Os}_{\mathrm{s}}$ & Beta 265098 & 12230 & 70 \\
\hline & unité G13 & AMS & Os & Beta 265097 & 12000 & 70 \\
\hline \multirow{12}{*}{$\begin{array}{c}\text { Pincevent } \\
\text { (La Grande-Paroisse ; Scinc-ct-Marnc) }\end{array}$} & nivcau IV20 & $\mathrm{CL} \Lambda$ & Charbon & Gif 6283 & 12120 & 130 \\
\hline & nivean TV20 & AMS & $\mathrm{Os}$ & OxA 148 & 12600 & 200 \\
\hline & niveau IV20 & AMS & Charbost & $0 \times \wedge 467$ & 12250 & 160 \\
\hline & niveau IV/20 & AMS & Charbon & Lill 37119 & 12450 & 45 \\
\hline & niveau TV213 & AMS & $\mathrm{Os}$ & OXA 149 & 12400 & 200 \\
\hline & nivcau IV213 & $\Lambda \mathrm{MS}$ & Os & $0 \mathrm{xA} 176$ & 12000 & 220 \\
\hline & niveau IV213 & AMS & $\mathrm{Os}$ & OxA 177 & 12300 & 220 \\
\hline & habitation $1 n^{\circ}$ ] & CLA & Charbon & Gill358 & 12300 & 400 \\
\hline & habitation $\mathrm{n}^{\circ} 1$ & $\mathrm{AMS}$ & Os & Lirl 6786 & 12277 & 96 \\
\hline & niveau TV30 & CIA & Charbon & Gir 6310 & 12100 & 130 \\
\hline & niveau IV/30 & AMS & Charbon & ETH 37120 & 12530 & 45 \\
\hline & niveau IV40 & CLA & Charbon & Gil 597I & 12100 & 120 \\
\hline \multirow{4}{*}{$\begin{array}{c}\text { Le Gramd-Canton } \\
\text { (Marolles-sur-Scine; Scine-ct-Marne) }\end{array}$} & niveáu supérieur & AMS & $\mathrm{Os}$ & Gif 9607 & 12080 & 155 \\
\hline & niveau supérieur & AMS & $\mathrm{Os}$ & OxA3139 & 12650 & 130 \\
\hline & niveau supérieur & AMS & $\mathrm{Os}$ & Gil 9608 & 12880 & 80 \\
\hline & niveau inférieur & AMS & $\mathrm{Os}$ & Gif 9606 & 12195 & 130 \\
\hline \multirow{4}{*}{$\begin{array}{c}\text { Le Tureau-des-Gardes } \\
\text { (Marolles-sur-Seine; Scinc-et-Marnc) }\end{array}$} & sectcur 6 & AMS & $\mathrm{Os}$ & Ly 6988 & 12290 & 90 \\
\hline & Secteur 10 & AMS & Os & AA 44214 & 12170 & 130 \\
\hline & secleur 10 & AMS & $\mathrm{Os}$ & AA 44215 & 12160 & 120 \\
\hline & secteur 10 & AMS & Os & AA 44216 & 12520 & 130 \\
\hline \multirow{2}{*}{$\begin{array}{c}\text { Le Tilloy } \\
\text { (Ville-Saint-Jacques ; Seine-ct-Marne) }\end{array}$} & mc̀tre C'15l & $\triangle \mathrm{AMS}$ & Os & $0 \mathrm{xA} 730$ & 12300 & 160 \\
\hline & mètre C.151 & AMS & $\mathrm{Os}$ & OxA 731 & 12240 & 160 \\
\hline $\begin{array}{c}\text { Le Brassot } \\
\text { (Étigny ; Yonne) }\end{array}$ & locus sud & AMS & Os & OxA 10096 & 12360 & 90 \\
\hline \multirow{4}{*}{$\begin{array}{l}\text { Le Buisson-Canpin } \\
\text { (Verberic ; Oise) }\end{array}$} & niveau Il.1 & AMS & Os & Gif $\wedge 95453$ & 12430 & 120 \\
\hline & niveau II.2 & AMS & Os & Gif A95454 & 12950 & 130 \\
\hline & niveau IJ..3 & AMS & $\mathrm{Os}$ & Gil'A99]06 & 12520 & 120 \\
\hline & nive:au TJ,3 & AMS & $\mathrm{Os}$ & Gif A99421 & 12300 & 120 \\
\hline \multirow{7}{*}{$\begin{array}{c}\text { Les Coudrays } \\
\text { (Ftiolles ; Fssone) }\end{array}$} & niveau Q-R5 & CLA & $O s$ & Ly 1351 & 12000 & 220 \\
\hline & unité N20 & AMS & $\mathrm{Os}$ & OXA 138 & 12900 & 300 \\
\hline & unitć N20 & AMS & Os & $0 \times \wedge 139$ & 13000 & 300 \\
\hline & unité N20 & $\mathrm{AMS}$ & Os & OXA 173 & 12800 & 220 \\
\hline & unité N20 & AMS & $\mathrm{Os}$ & OxA 175 & 12900 & 220 \\
\hline & locus 1 & AMS & Os & OxA 5995 & 12250 & 100 \\
\hline & locus 2 & AMS & Os & $0 \times \wedge 8757$ & 12313 & 75 \\
\hline $\begin{array}{c}\text { La Côte-Masset } \\
\text { (Bonnic̀res-sur-Scinc ; Yvclines) }\end{array}$ & nive:alu 4 & AMS & Os & Gif A93014 & 12770 & 120 \\
\hline
\end{tabular}

Tabl. 1 - Liste des dates ${ }^{14} \mathrm{C}$ obtenues sur les gisements magdaléniens du Bassin parisien (exception faite de la Haye et de la Côte-Masset, les données sont issues d'un inventaire réalisé par P. Bodu et paru en annexe du rapport 2004 du projet collectif de recherche «Habitats et peuplements tardiglaciaires du Bassin parisien », Nanterre - Saint-Denis, UMR 7041-SRA d'Île-deFrance).

Table 1 - List of ${ }^{14} \mathrm{C}$ dates yield by Magdalenian sites in the Paris Basin (except those for La Haye and Côte-Masset, these data were collected by P. Bodu and featured in appendices of the 2004 report of the Projet collectif de recherche "Habitats et peuplements tardiglaciaires du Bassin parisien", Nanterre - SaintDenis, UMR 7041-SRA d'île-de-France). 


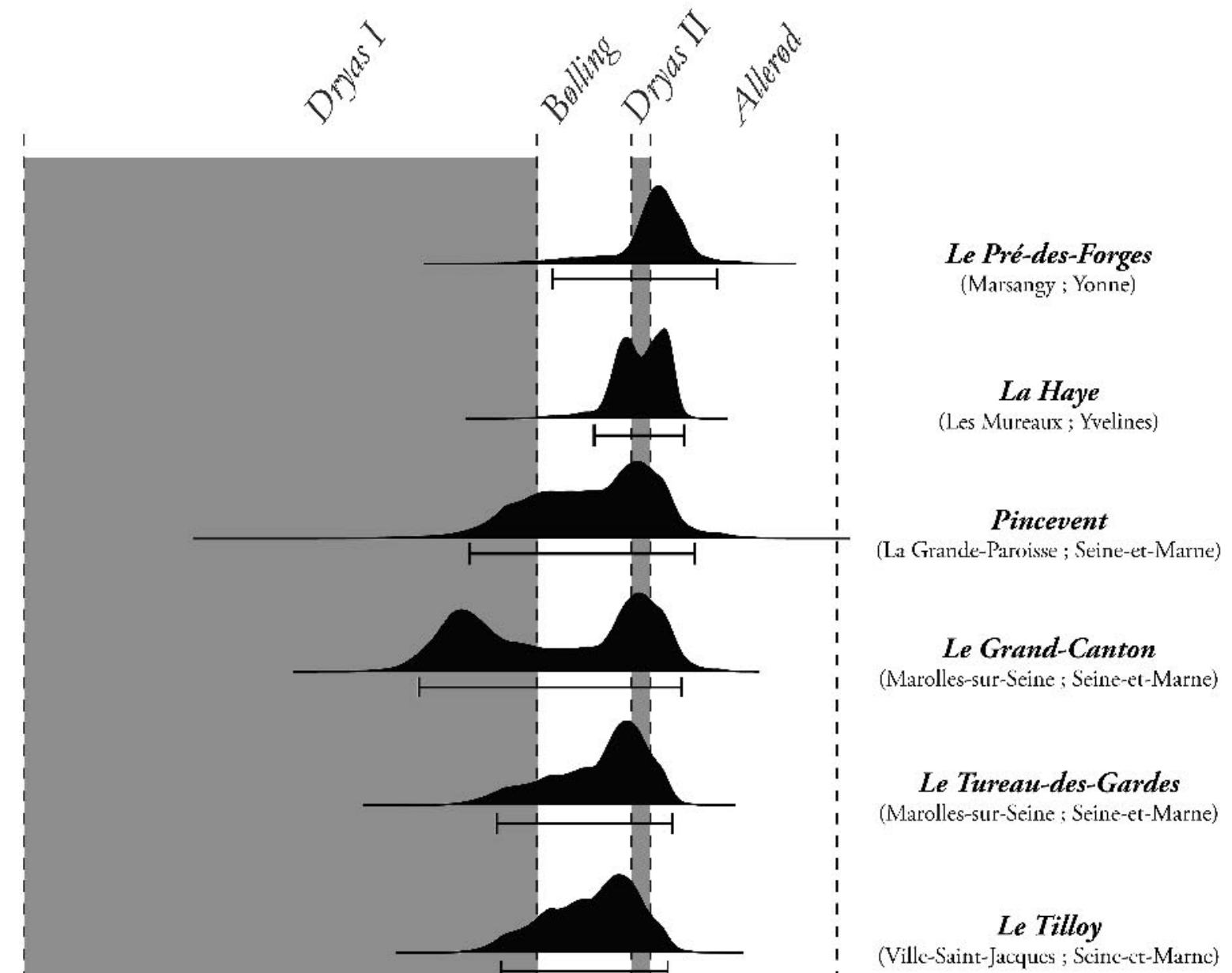

(Villc-Saint-Jacques; Scinc-ct-Marnc)

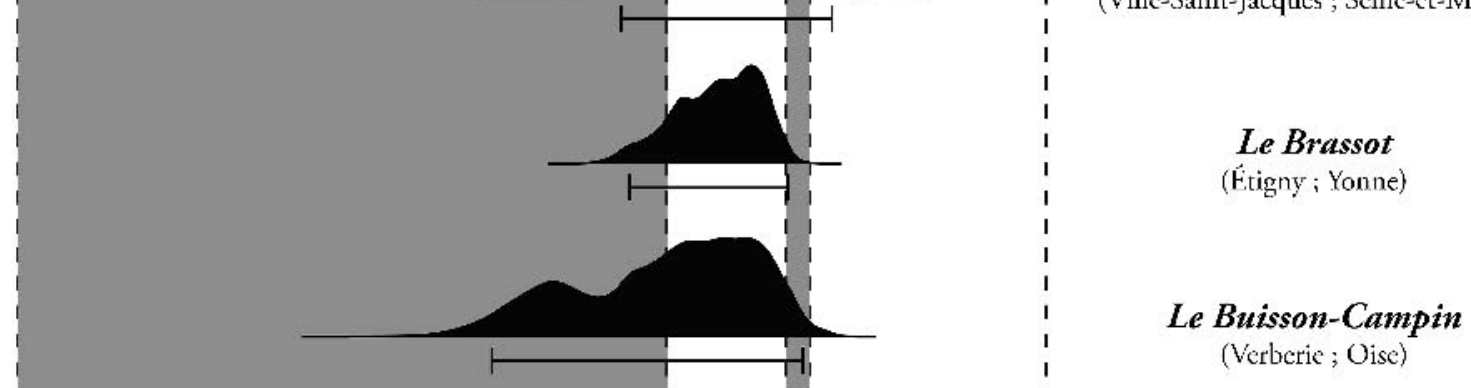

\section{Les Coudrays}

(Ériolles; Essone)

\section{La Côte-Masset}

(Bonnières-sur-Seine ; Yelines)

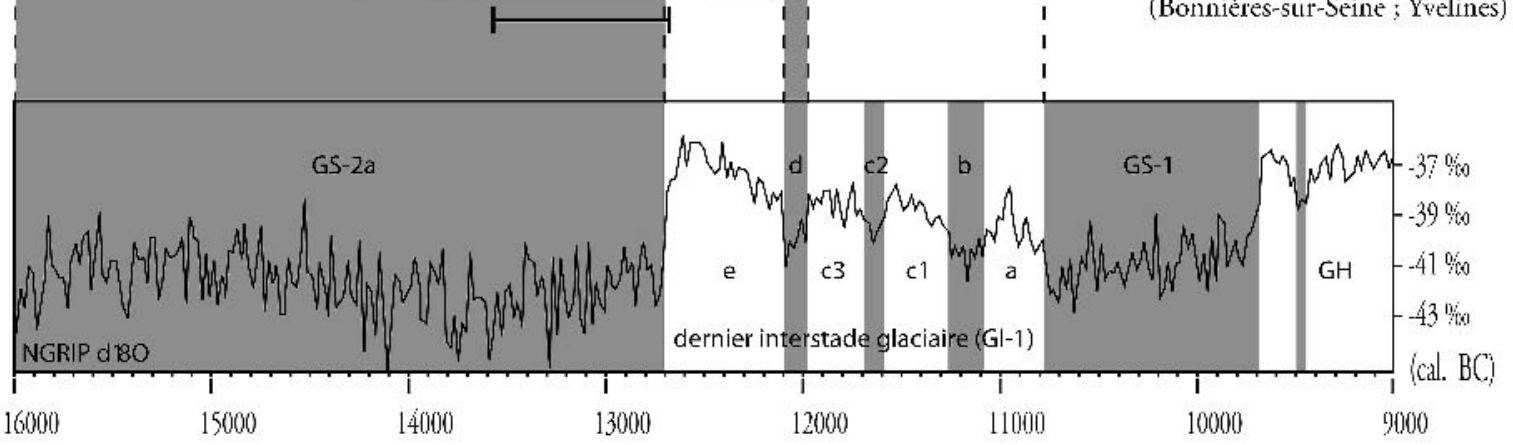

Fig. 10 - Calibration des âges ${ }^{14} \mathrm{C}$ obtenus sur les gisements magdaléniens du Bassin parisien (au moyen du logiciel Calib 5.0.2 intégrant les résultats de la courbe IntCal04; courbe des variations isotopiques de $1^{1}{ }^{18} \mathrm{O}$ d'après les carottes NGRIP du Groenland, GICC05; voir notamment Rasmussen et al., 2006). Fig. $10-{ }^{14} \mathrm{C}$ age calibration of Paris Basin Magdalenian sites (with Calib 5.0.2 using IntCal04 curve; ${ }^{18} \mathrm{O}$ isotopic variations of Greenland NGRIP and GICC05 records; see particularly Rasmussen et al., 2006). 
séquences naturelles régionales et d'apporter de précieuses informations sur l'environnement végétal, encore peu connu, au sein duquel les populations magdaléniennes régionales ont installé leurs habitats.

\section{DES ACTIVITÉS ET UN ÉQUIPEMENT TECHNIQUE FIDÈLES AUX TRADITIONS RÉGIONALES}

Nous l'annoncions plus haut, seule une petite partie des vestiges de la concentration principale a été prélevée. Il s'agit, pour l'essentiel, d'ossements manifestement trop fragiles pour être laissés en place lors du rebouchage et d'objets en silex déplacés par la pelle mécanique lors des opérations de sondage. S'y ajoute le crâne de cheval découvert à une centaine de mètres de la concentration principale. L'analyse qui suit s'appuie donc sur ces quelques vestiges prélevés et sur l'observation préliminaire des vestiges laissés en place.

\section{Une chasse (spécialisée?) au renne}

L'un des principaux intérêts du niveau d'occupation de la Haye réside dans la présence de nombreux témoins osseux. Leur surface, bien que souvent vermiculée, ne paraît que faiblement altérée par les phénomènes physico-chimiques liés au weathering. Ce bon état de conservation général conforte l'hypothèse d'un enfouissement rapide des vestiges. La découverte de plusieurs tarses de renne en connexion anatomique et la présence d'éléments squelettiques particulièrement sensibles à la «fonte taphonomique», comme le bois de cervidé, les côtes et les vertèbres, confirment des conditions de conservation exceptionnelles et témoignent de l'absence de perturbations postdépositionelles. On observe toutefois un gradient dans le degré de préservation selon un axe nord-est - sud-ouest. Dans la partie nord, le caractère plus sableux du sédiment encaissant s'est logiquement révélé moins favorable à la conservation des vestiges organiques. Tous les restes osseux ont fait l'objet d'une consolidation sur place au moyen d'un agent consolidant (Rhodopas® M) dilué dans l'acétone à environ $10 \%$.

Au total, 76 restes osseux (tabl. 2) ont été prélevés. Les observations effectuées au cours des étapes de fouille et de consolidation tendent à montrer que ces éléments, souvent décomposés en plusieurs fragments, sont relativement représentatifs de l'ensemble de ceux laissés en place sur le terrain. Près de la moitié d'entre eux $(48 \%)$ a pu être déterminée. La presque totalité (97\%) se rattache au renne. Voilà une représentation qui n'est pas sans rappeler celle de certaines occupations de Pincevent et de Verberie (David, 1994). Bien que tentant, il serait toutefois un peu précipité d'inscrire d'ores et déjà l'occupation de la Haye dans le groupe des habitats automnaux accueillant les épisodes de chasse spécialisée au renne. Pour l'heure, il faut se contenter de préciser que plusieurs parties du squelette de ce taxon ont pu être identifiées (fig. 11). La tête est représentée par plusieurs séries dentaires, mais aussi par des fragments de ramure et par un bois de massacre entier (fig. 12). La cage thoracique et le rachis sont, pour leur part, représentés par un ensemble de côtes bien conservées et par deux fragments interprétés comme de possibles éléments de vertèbres. Les membres antérieurs et postérieurs sont attestés par des fragments de radius, de fémur et de tibia, ainsi que par quelques phalanges.

Une première estimation s'appuyant sur les séries dentaires et certaines parties du membre antérieur (les fragments de radius notamment) fixe pour l'instant à deux le nombre minimum d'individus apportés dans l'occupation. On distingue un renne adulte âgé de 7 à 10 ans et un jeune adulte âgé de 3 à 4 ans. L'absence de dents appartenant à de très jeunes individus n'offre pas, pour le moment, la possibilité de déterminer la saison d'occupation du site. La présence d'un bois de massacre, plutôt de grande taille, provenant vraisemblablement d'un individu mâle permettrait toutefois de situer l'abattage de cet individu entre le mois de septembre et la mi-décembre. L'occupation se placerait donc entre la fin de l'été et le début de l'hiver, à moins de considérer que les bois de massacre ont été transportés pendant plusieurs mois comme réserve de matière première.

Outre les nombreux restes de renne - taxon qui, précisons-le, constitue avec le cheval les deux proies de prédilection des Magdaléniens du Bassin parisien (Bignon, 2007) -, on note la présence d'un fragment de diaphyse dont les dimensions suggèrent plutôt une appartenance à un équidé ou à un boviné. Enfin s'ajoute le crâne de cheval que l'on a déjà évoqué à diverses reprises (fig. 3). Il provient d'un individu âgé de 5 à 6 ans et comprend l'arcade des incisives, les séries de dents jugales supérieures (molaires et prémolaires), ainsi que l'os du palais. L'absence de canines sur cet individu

\begin{tabular}{|c|c|c|}
\hline Partie squelettique & Nombre de restes & \\
\hline Radius et ulna & 1 & \\
\hline Dent & 2 & \\
\hline Humérus & 1 & \\
\hline Côte & 9 & \\
\hline Diaphyse & 12 & \\
\hline Tibia & 2 & \\
\hline Phalange & 10 & \\
\hline Vertèbre & 2 & \\
\hline Os plat & 1 & \\
\hline Bois de renne & 3 & \\
\hline Phalange et diaphyse & 1 & \\
\hline Radius & 2 & \\
\hline Grand cunéiforme & 1 & \\
\hline Fémur & 1 & \\
\hline Métacarpe & 1 & \\
\hline Indéterminé & 27 & \\
\hline TOTAL & 76 & \\
\hline Taxon & NR & $\%$ NRD \\
\hline Renne & 36 & 97,3 \\
\hline Équidé/Boviné & 1 & 2,7 \\
\hline Sous-total restes déterminés & 37 & 100 \\
\hline Restes indéterminés & 39 & \\
\hline TOTAL & 76 & \\
\hline
\end{tabular}

Tabl. 2 - Décompte du nombre de restes osseux prélevés au cours de la fouille.

Table 2 - Breakdown of bone remains collected during the excavations. 


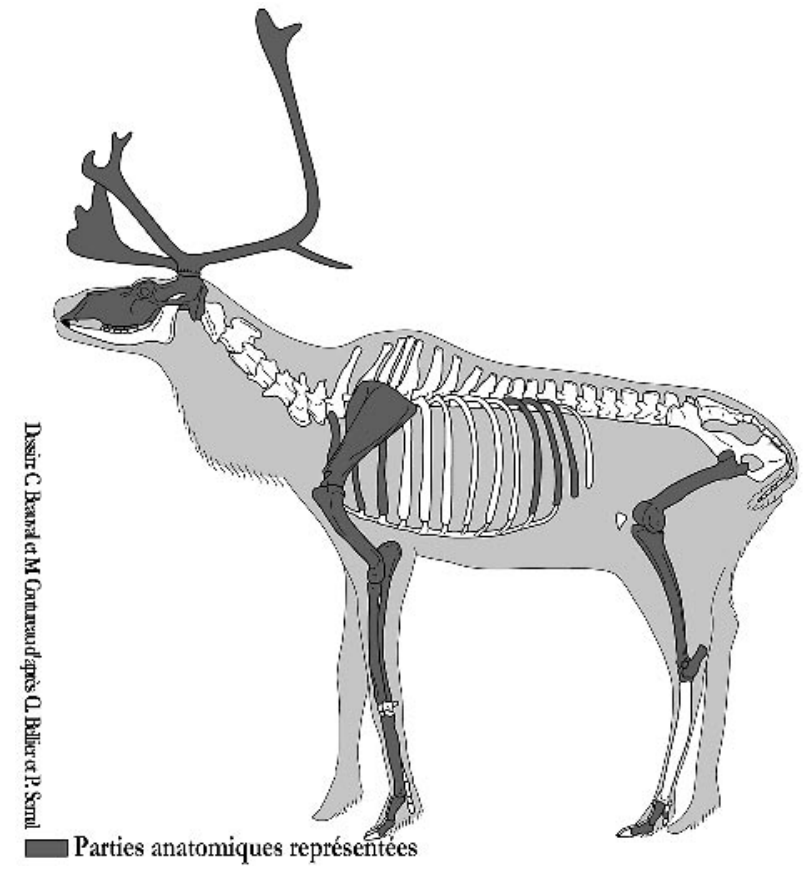

Fig. 11 - Renne : parties squelettiques représentées. Fig. 11 - Reindeer skeletal elements observed.

relativement âgé indique qu'il s'agit d'une femelle. Aucun autre vestige n'a été repéré dans l'un des nombreux sondages ouverts aux alentours de ce crâne. L'absence de la mandibule pourrait signifier qu'il s'agit d'un rejet anthropique effectué à la suite d'une opération de boucherie. Toutefois, on ne peut pas non plus exclure totalement que cet élément se trouve en position secondaire après un déplacement postdépositionnel.

\section{L'industrie lithique}

Ce sont 242 objets en silex qui ont été prélevés lors des opérations de terrain. La quasi-totalité provient de la concentration principale. Il s'agit essentiellement d'éclats et d'esquilles, mais on y observe aussi un nucléus (fig. 13, $\mathrm{n}^{\mathrm{O}}$ 3), un fragment de lame, un fragment de lamelle, ainsi que deux outils (fig. 13, $\mathrm{n}^{\text {os }} 1$ et 2). Les éclats, le fragment de lame, le nucléus et l'un des deux outils correspondent au lot de pièces qui a été déplacé par le godet de la pelle mécanique le jour de la découverte du site. Le second outil a été découvert en marge de la concentration, à plus de 10 mètres à l'ouest du foyer. Il s'agit d'un burin dièdre aménagé sur la partie proximale d'une lame qui présente un bord ocré sur la presque totalité de sa longueur (fig. 13, $\mathrm{n}^{\mathrm{O}} 2$ ). La majorité des esquilles a été relevée et démontée lors de la fouille du foyer et des deux amas de débitage qui le jouxtent.

L'essentiel des matières premières exploitées par les occupants est un silex du Crétacé supérieur, probablement issu des étages campanien et santonien. La surface émoussée, voire roulée, du cortex de certains objets évoque un ramassage de blocs en position secondaire. Les nodules ont donc été plus vraisemblablement

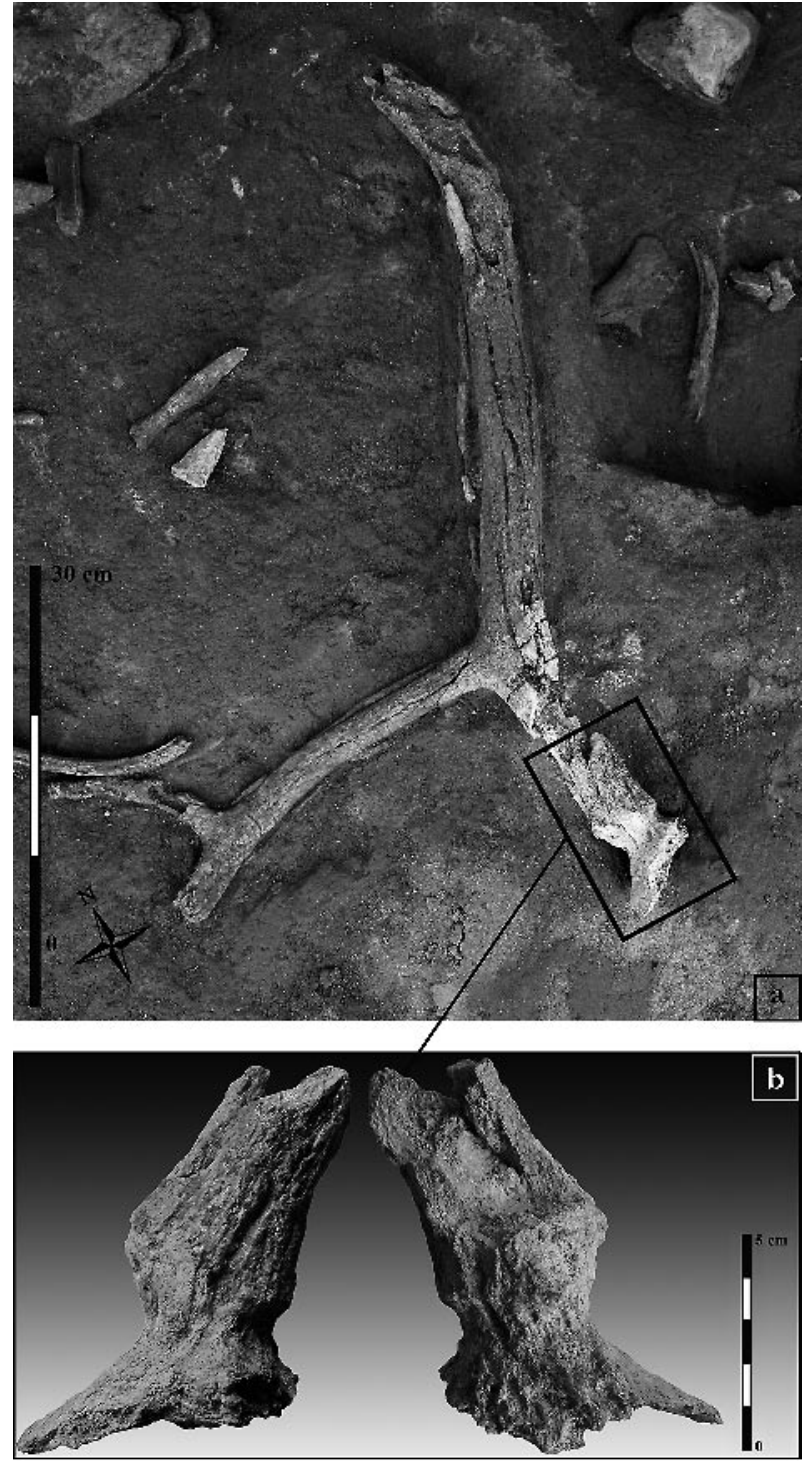

Fig. 12 - Bois de massacre de renne.

Fig. 12 - Antler from a hunted reindeer.

collectés sur les berges de la Seine que prélevés en position primaire au sein de bancs en place qui auraient été accessibles localement. On relève en outre la présence d'un matériau dont l'origine géologique est assurément tertiaire. Il rappelle pour beaucoup le silex rouge provenant de l'étage ludien de l'Éocène supérieur que l'on rencontre sous la forme de produits importés dans plusieurs autres occupations magdaléniennes de la région (Mauger, 1994). Sur l'occupation de la Haye, ce matériau est représenté par un outil double burin-grattoir (fig. $13, \mathrm{n}^{\mathrm{0}} 1$ ) et par plusieurs lames regroupées près d'une grande dalle en grès ocrée (fig. 14). Signalons également la présence d'une lamelle en silex tertiaire de couleur brun moucheté de blanc qu'il faut bien différencier du matériau ludien évoqué précédemment.

Parmi les vestiges conservés in situ, nous avons pu identifier plusieurs autres burins et, dans une moindre quantité, des grattoirs et des perçoirs. En ce qui concerne les armatures, quelques lamelles à dos se trouvaient mêlées aux déchets de débitage jouxtant la 
bordure nord-ouest du foyer. L'une des trois pièces présente un dos aménagé par retouche inverse. Bien qu'anecdotique, cette indication renforce la cohérence qui se dégage de quelques comparaisons (au niveau de la production de lames et de lamelles notamment) avec d'autres industries du Magdalénien supérieur régional (Schmider, 1971; Valentin, 1995 ; Debout, 2003).

\section{Une pointe de sagaie en bois de renne}

Une pointe de sagaie en bois de renne figure dans le lot des objets qui ont été récupérés avant le re- bouchage du sondage (fig. 15). Découverte à moins de 2 mètres de la bordure ouest du foyer, elle est longue de 78 millimètres, mais présente en partie distale une cassure dont il est encore difficile de dire si elle résulte de la fabrication ou de l'utilisation. De section subrectangulaire, la largeur et l'épaisseur ont été respectivement mesurées à 14 millimètres et 9 millimètres. Un biseau double d'une longueur de 24 millimètres a été aménagé sur les grandes faces. L'objet semble avoir été réalisé à partir d'une baguette de bois de renne. À cet égard, il faut signaler la présence, à quelques centimètres, d'un grand fragment de perche qui pourrait porter des traces de débitage (fig. 16).
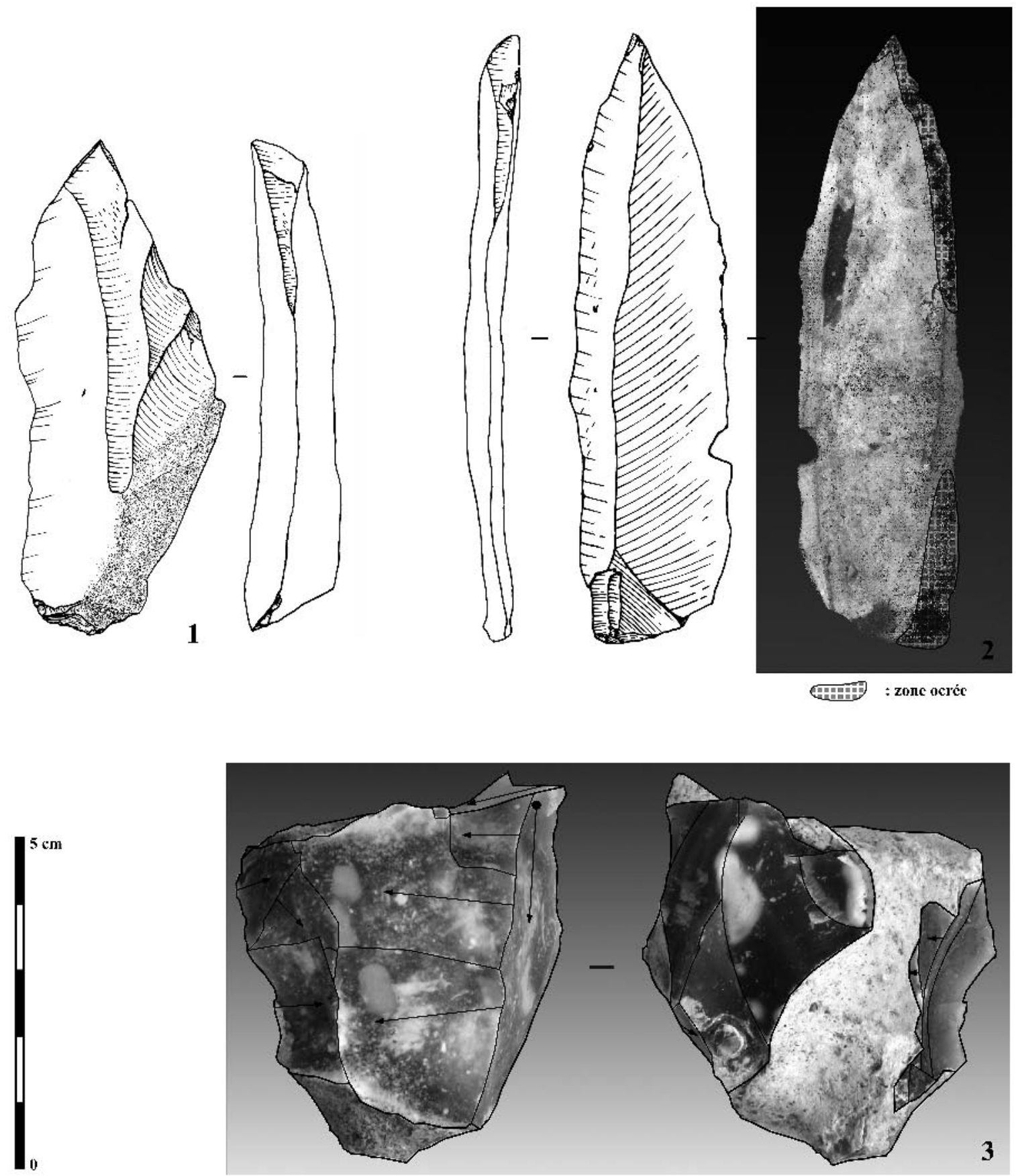

Fig. 13 - Quelques exemples d'objets en silex qui ont été prélevés.

Fig. 13 - Some knapped flint artefacts collected during excavations. 
Bien que la surface soit fortement vermiculée, on peut distinguer une rainure longitudinale incisée sur près de la moitié de la longueur de la pièce. Cette pointe s'inscrit indubitablement dans la tendance typologique dominante reconnue dans les séries magdaléniennes du Bassin parisien (Pétillon, 2008). Ne manque pour être entièrement typique que la série de stries sur le biseau, que l'on observe sur certains exemplaires régionaux. Signalons enfin que les pointes de sagaies osseuses sont rares dans le Magdalénien du Bassin parisien, comme dans tous les sites de plein air. Pour l'heure, un récent inventaire fait état d'un

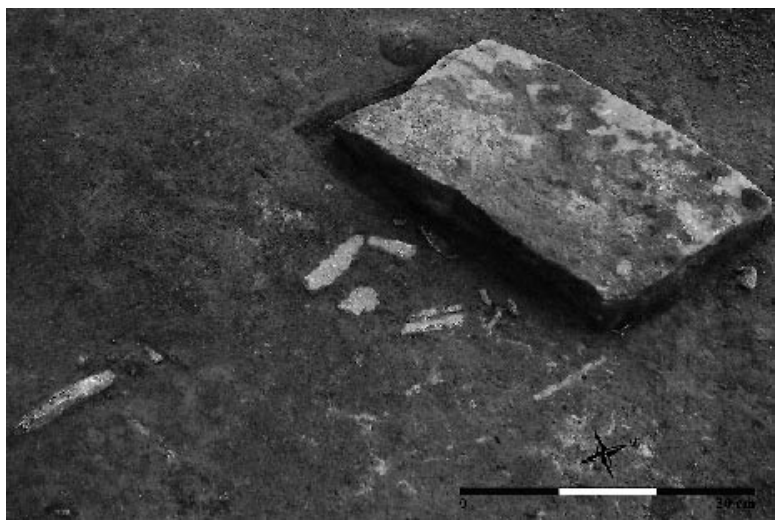

Fig. 14 - Plusieurs lames situées à proximité d'une grande dalle ocrée en H15.

Fig. 14 - Several blades located close to a large ochred stone in H15. total de 48 exemplaires (Pétillon, 2008). L'étendue estimée de l'occupation de la Haye laisse augurer d'autres découvertes qui permettront d'étoffer le corpus régional.

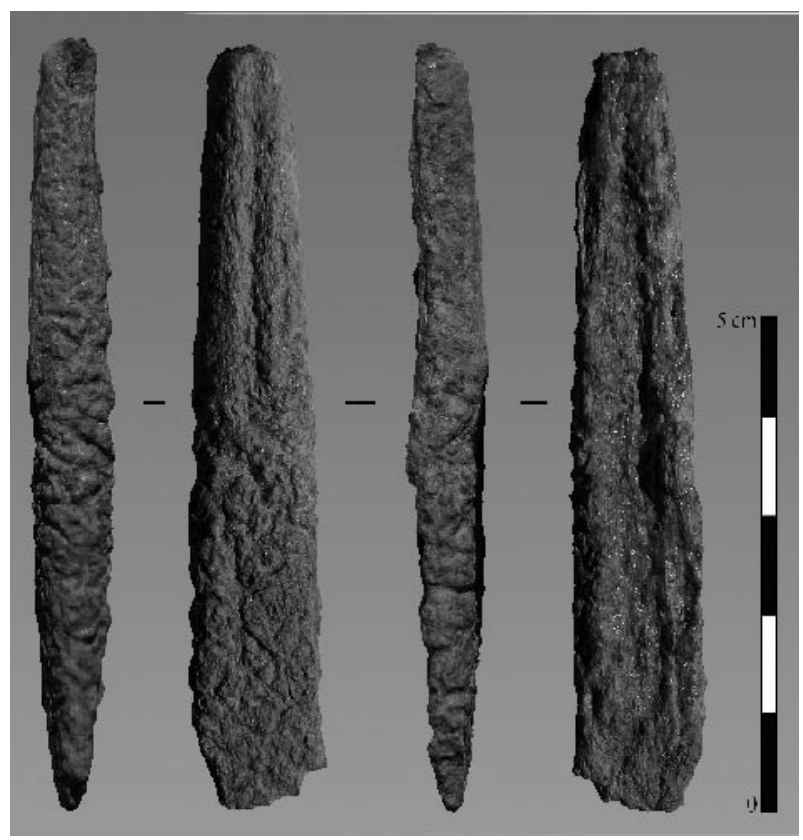

Fig. 15 - Pointe de sagaie en bois de renne. Fig. 15 - Reindeer antler point.

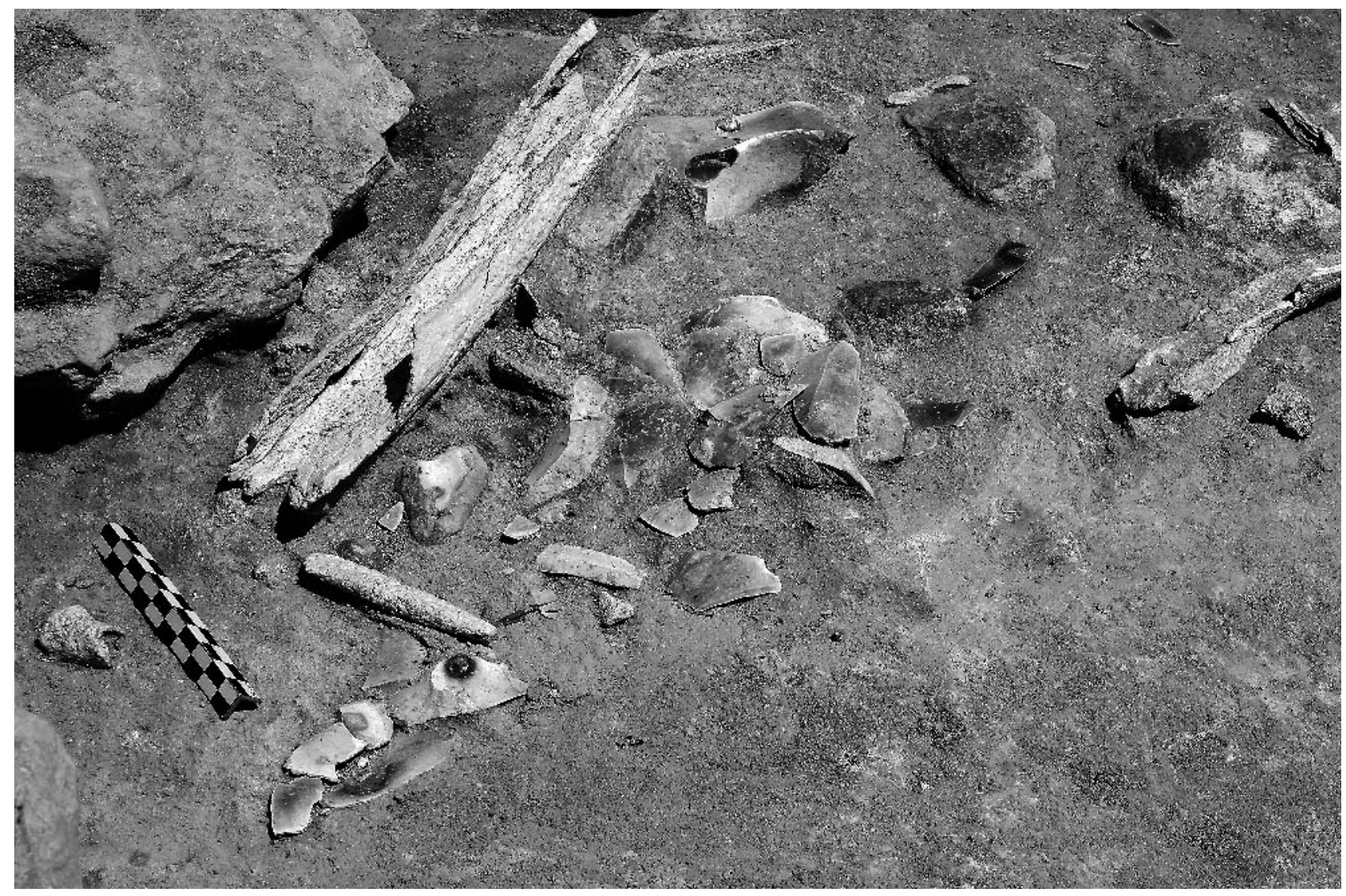

Fig. 16 - Secteur dans lequel a été découverte la pointe de sagaie.

Fig. 16 - Sector where the reindeer antler point was found. 


\section{Un indice d'activités symboliques}

Un fragment de fossile d'un gastéropode marin a été recueilli au sein d'un petit amas de restes de taille dégagé en bordure est du foyer (fig. 17). Il s'agit d'un moule interne, conservé sur une longueur inférieure à 2 centimètres, appartenant au genre Haustator, de la famille des Turritellidae, abondant dans les terrains d'âge éocène moyen et largement répandu dans toute l'Europe occidentale. Habituellement interprétés comme des éléments de parure, les coquillages tertiaires sont fréquemment rencontrés dans les habitats magdaléniens régionaux (Taborin, 1994), mais dans des proportions variables et cela même d'un niveau à l'autre d'un même gisement (Vanhaeren, 2006). Ils ne sont pas pour autant porteurs d'un indice chronologique sûr dans la mesure où l'on en rencontre de nombreux exemplaires dans des contextes archéologiques plus anciens (Bodu et al., 2005). Comme il s'agit du moule interne, et non de la coquille, un doute subsiste sur l'utilisation de l'exemplaire que nous avons récolté en tant d'élément de parure. Il faut toutefois souligner qu'il est empreint d'ocre, ce qui suggère une certaine implication de cet objet au sein des activités réalisées par les occupants.

\section{Une variété de roches non taillées pour différents usages}

Toutes les roches ayant été conservées in situ, les indications préliminaires concernant cette catégorie de vestige reposent uniquement sur des observations de terrain. Les roches dégagées sur le sol d'habitat et dans le foyer se caractérisent par une diversité pétrographique et morphologique. Elles comprennent essentiellement des grès, des grès schisteux et des calcaires. La plupart présentent des surfaces corticales roulées suggérant plutôt un approvisionnement sur les rives de la Seine. En complément des roches contenues dans le foyer, vraisemblablement réservées à son fonctionnement, quelques autres blocs de grandes dimensions pourraient avoir servi comme éléments d'infrastructure ou de calage, compte tenu de leur taille et de leur poids. C'est notamment le cas de plusieurs calcaires volumineux, sans traces de feu apparentes, situés à proximité immédiate du foyer G13. De tels éléments sont connus dans d'autres habitations magdaléniennes du Bassin parisien, où ils délimitent parfois un cercle de plusieurs mètres de diamètre (Julien, 1988).

Les grès schisteux semblent avoir été intégrés à des activités mettant en jeu l'ocre rouge. Sur les trois plus gros volumes découverts, des traces de colorant rouge ont en effet été décelées sur au moins une des faces. Il en va de même pour certains petits fragments de fines plaquettes de schiste localisés à l'est du foyer. Ces éléments ont probablement servi à réduire le colorant en poudre et sont donc à classer dans la catégorie des objets domestiques. Des exemplaires comparables ont récemment été découverts à Pincevent (Julien et Beyries, 2006). La fonction de support à broyer le colorant est d'autant plus probable pour les éléments de la Haye que les matières colorantes rouges y sont bien repré- sentées et forment une grande nappe qui s'étend au nord du foyer G13 (voir ci-dessous).

\begin{tabular}{c}
\hline PREMIERS ÉLÉMENTS \\
D'ORGANISATION SPATIALE \\
\hline
\end{tabular}

\section{Structuration et fonction de l'aire d'occupation principale}

La lecture et l'interprétation de la structuration spatiale des quelques mètres carrés de sol d'habitat dégagés à la Haye sont facilitées par une densité relativement faible de vestiges (fig. 17). Ceux-ci s'articulent autour d'un foyer à cuvette implanté en G13 (fig. 18) qui a manifestement polarisé la majeure partie des activités. Un autre foyer pourrait se situer en G15, à l'emplacement d'une grande dalle profondément ancrée dans le sédiment encaissant et qui suggère l'emplacement d'une cuvette. De plus, le sédiment tendait à se charger en charbons au fur et à mesure de la progression du décapage que nous avons toutefois rapidement interrompu afin de ne pas fragiliser cet emplacement.

Une petite cuvette a été repérée en $\mathrm{H} 14$ à proximité du foyer G13. D'un diamètre irrégulier de 25 centimètres et d'une profondeur d'une dizaine de centimètres, elle était comblée par du sédiment sableux et contenait quelques produits de débitage de silex. À l'instar du sol environnant, le fond et les parois étaient ocrés. Dans l'état actuel de la documentation, il est impossible de déterminer s'il s'agit d'une structure naturelle ou d'une fosse creusée intentionnellement.

Par ailleurs, on relève l'aspect dissymétrique de la répartition des témoins osseux par rapport à l'implantation du foyer G13 (fig. 19) : relativement nombreux au sud de la structure de combustion, ils sont pratiquement absents au nord du foyer. La nature plus sableuse du sédiment dans la partie nord ne suffit pas à expliquer leur absence dans la nappe d'ocre qui se développe au nord du foyer à cuvette. Cette répartition différentielle témoigne donc vraisemblablement d'un comportement de rejet systématique de la part des occupants.

Deux postes de débitage sont situés sur la bordure nord-est du foyer G13 (fig. 18). D'après les observations réalisées lors de la fouille, il s'agit de restes de taille issus de deux opérations de débitage destinées à la production de lamelles. Pour la concentration dégagée en $\mathrm{H} / 13-14$, un nucléus est resté au contact des déchets de taille. Il s'agit d'un gros éclat support qui semble avoir été mis en forme par l'aménagement d'une crête. Deux autres concentrations de restes de débitage semblent plutôt correspondre à des amas de rejet. Celle située en F-G/13, à moins de 1 mètre de la bordure sud du foyer G13, se trouve dans une zone charbonneuse qui pourrait matérialiser l'emplacement d'une aire de rejet de produits de combustion évacués de la cuvette lors d'un réaménagement de la structure. La concentration repérée en D-E/15-16, à un peu plus de 3 mètres au sud-est du foyer G13, n'a été dégagée que sommairement, mais semble essentiellement contenir les restes d'une opération de débitage ayant produit des lames, puis des lamelles. La nature exacte de ces 
deux concentrations ne pourra toutefois être établie que par le remontage des déchets de taille et l'analyse de la distribution spatiale des produits. Enfin, on peut signaler le regroupement de quelques supports laminaires en silex tertiaire à proximité d'une grande dalle en H15. L'ensemble pourrait être interprété comme un espace de réserve de supports non utilisés (fig. 14).
La nappe d'ocre rouge, bien visible lors de la fouille, s'étend sur un peu plus de 2 mètres carrés au nord-est du foyer à cuvette. Cette dernière et l'aire de rejet des restes osseux se retrouvent symétriquement opposées par rapport au foyer. Cette configuration renvoie au schéma théorique qu'A. Leroi-Gourhan avait élaboré pour des unités d'occupation magdaléniennes à partir de l'analyse

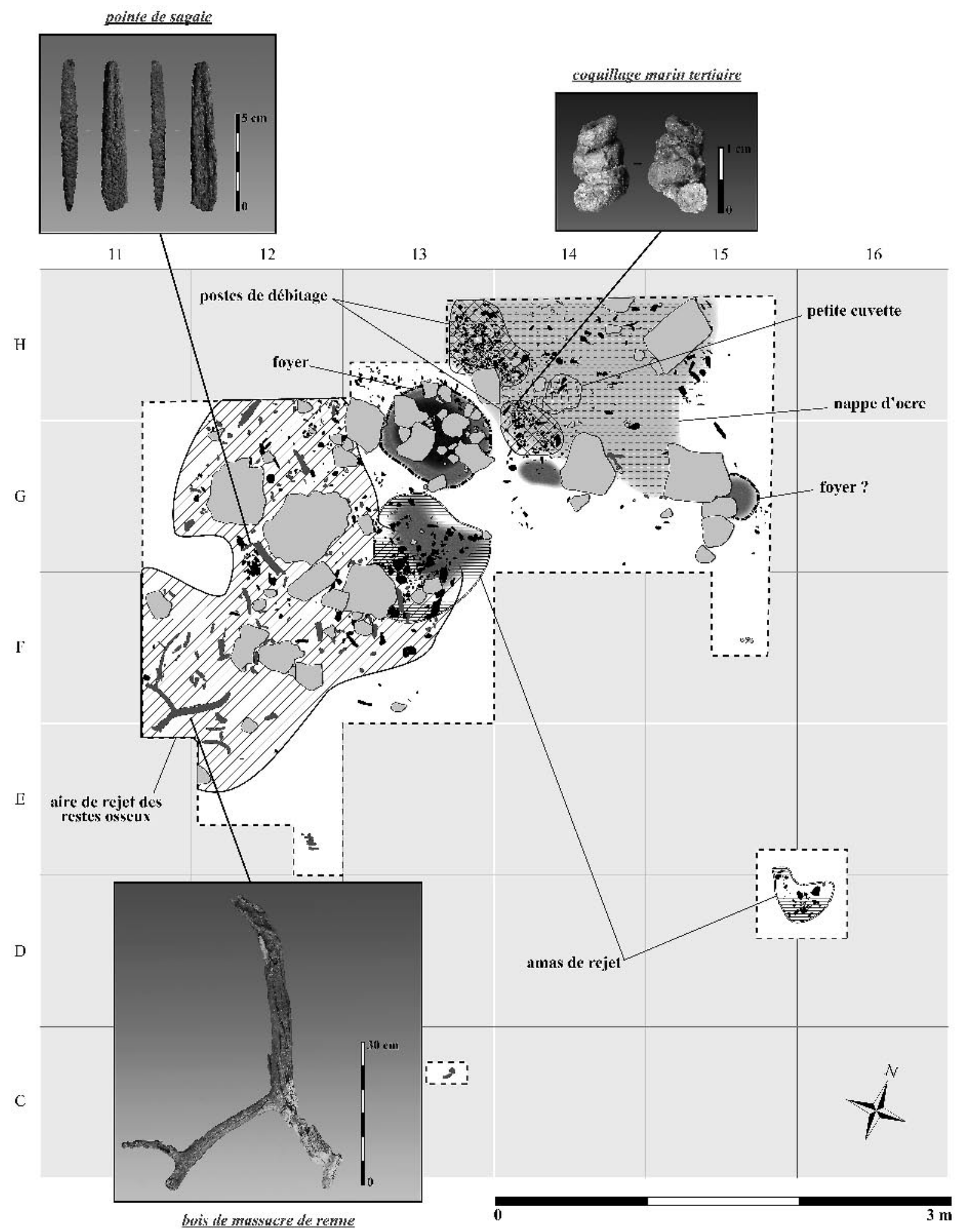

Fig. 17 - Plan interprétatif du niveau d'occupation magdalénien.

Fig. 17 - Interpretative map of the Magdalenian horizon occupation unit. 


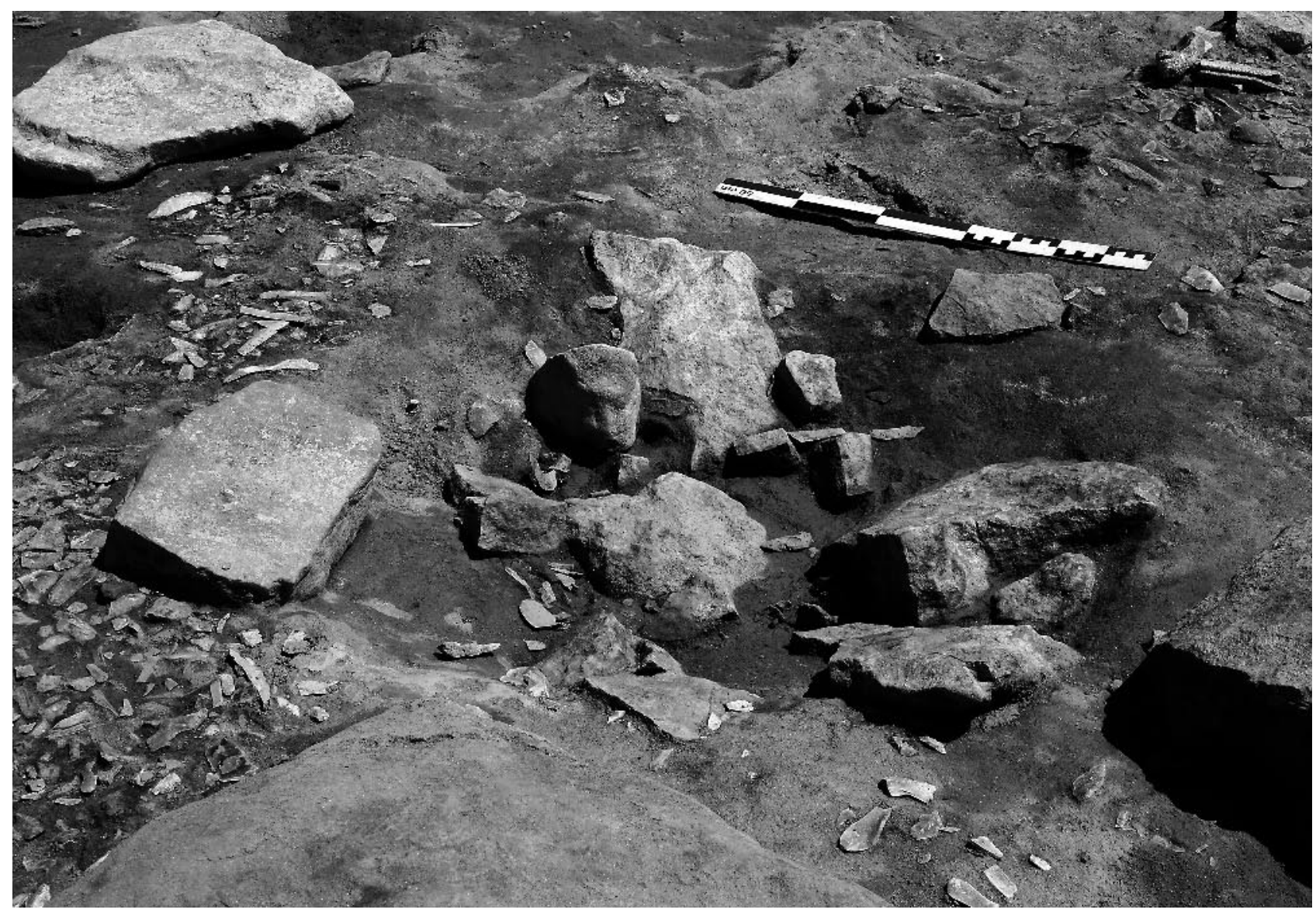

Fig. 18 - Le foyer à cuvette G13.

Fig. 18 - The pit hearth G13.

spatiale de plusieurs niveaux d'habitat magdaléniens de Pincevent (Leroi-Gourhan et Brézillon, 1972). Bien que ne couvrant qu'une dizaine de mètres carrés, la portion de sol d'habitat qui a été dégagée à la Haye offre de nombreux points de comparaison avec d'autres «unités d'occupation» analysées dans le Bassin parisien. Pour illustrer ces similitudes, nous avons choisi l'exemple de l'unité J 116 du niveau IV40 de Pincevent (fig. 20), l'une des occupations les mieux conservées de la séquence de ce gisement (Debout, 2007). Outre les concordances dans la configuration générale de l'organisation des vestiges des deux sols d'habitat, on constate des densités de matériel comparables, sans doute liées à des occupations de courte durée. Cette confrontation renseigne par ailleurs sur l'étendue possible de l'occupation de la Haye. Il est en effet probable qu'elle se développe sur au moins 100 mètres carrés, mais cette surface pourrait être plus étendue si des structures annexes (petit foyer complémentaire de celui ou de ceux localisés au centre de l'occupation, amas de rejet ou poste(s) de débitage périphériques, etc.) telles que l'on en rencontre dans la plupart des occupations de Pincevent (Bodu, 1993; Julien et Karlin, 2002) s'y ajoutaient.

Parmi les témoins d'activités, ceux en relation avec la chasse sont relativement bien représentés (ossements, armatures en silex, extrémité de projectile en matière dure animale, etc.). L'identification des parties squelettiques des animaux abattus indique que deux rennes au moins ont été apportés aux abords du foyer à cuvette. On y trouve non seulement les parties riches en viande, mais aussi celles qui le sont moins, comme le bas des pattes. Un fragment de diaphyse appartenant probablement à un boviné ou à un équidé témoigne aussi de l'apport de certaines parties squelettiques d'un ongulé de plus grande taille. La consommation des matières animales occupe donc une part importante des activités menées autour du foyer G13. Les activités techniques sont également bien représentées. Elles concernent notamment l'entretien des armes de chasse, matérialisé par le rejet d'une pointe de sagaie brisée, des lamelles à dos usagées, des déchets de taille témoignant de la production de supports lamellaires en vue du remplacement des armatures rejetées et des déchets d'exploitation d'un bois de renne. Le traitement des peaux est par ailleurs suggéré par la présence d'au moins un grattoir et par celle des plaques de grès ayant servi au broyage de l'ocre. Dans l'état actuel, il est impossible de déterminer si cette aire d'activité centrée sur un foyer à cuvette était associée à une structure d'habitation. Dans les sites magdaléniens du Bassin parisien, il est courant de distinguer deux types d'unités d'occupation: les unités d'habitation et les unités annexes. Si l'on se reporte à la définition proposée pour ces deux types (Olive et al., 2000), la concentration de vestiges associée au foyer G13 de la Haye se rattache 


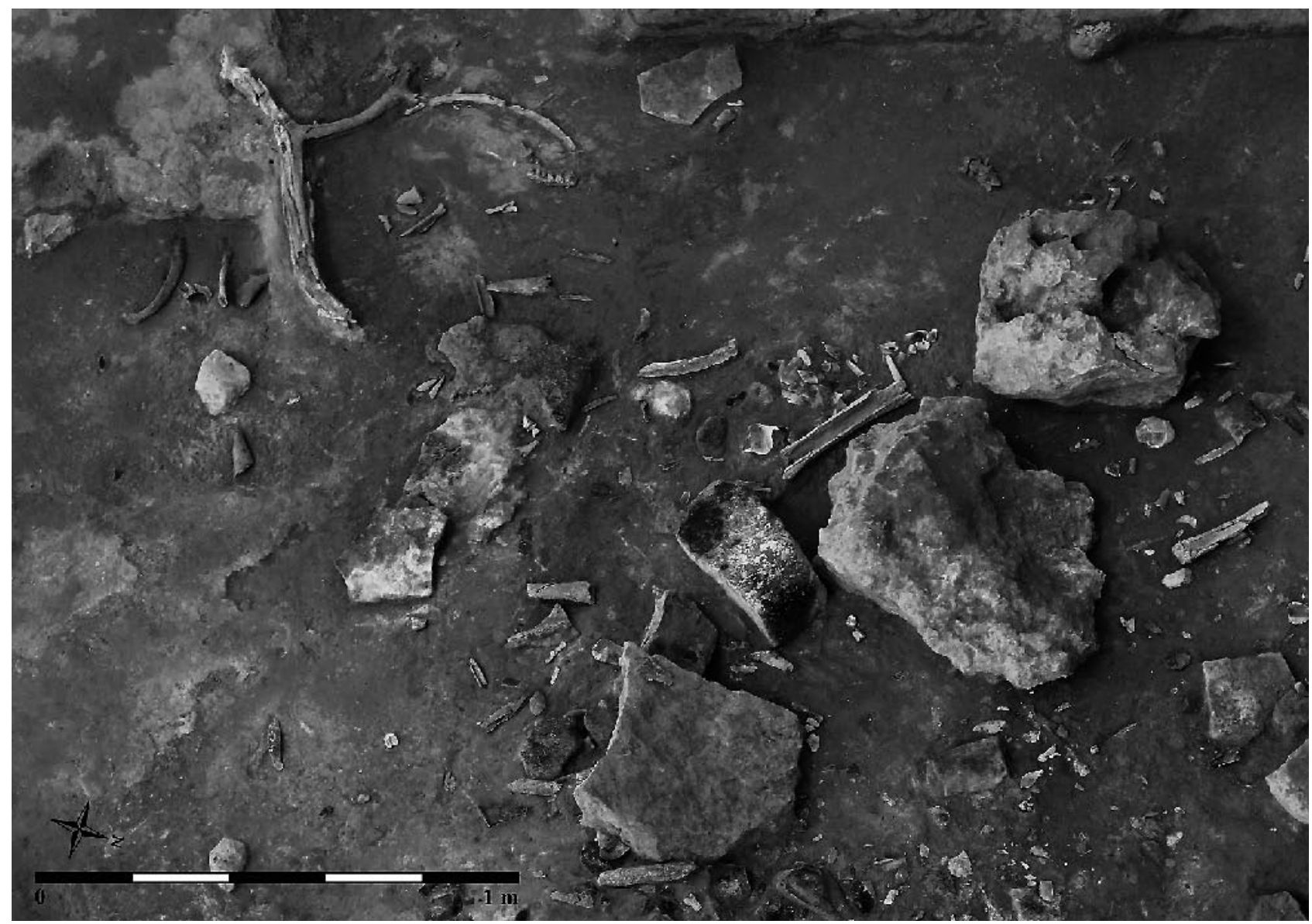

Fig. 19 - Aire de rejet des restes osseux qui s'étend au sud du foyer G13.

Fig. 19 - Concentration of bone remains to the south of the pit hearth G13.

plutôt au premier type. La présence de nombreux vestiges osseux et la possibilité d'appliquer le modèle théorique d'habitation élaboré par A. Leroi-Gourhan constituent les plus solides arguments en faveur de cette proposition. La question doit néanmoins rester ouverte tant que la surface de fouille n'aura pas été élargie.

\section{La structure pierreuse de la zone ouest}

La structure pierreuse qui a été dégagée dans la zone ouest du gisement, à environ 500 mètres du niveau d'habitat magdalénien décrit précédemment, s'étendait globalement sur une surface ovale de $2,70 \mathrm{~m}$ de longueur et de 1,50 m de largeur (fig. 21). Elle se composait essentiellement de blocs de calcaire et de grès ne montrant aucune trace de feu (souvent de type glauconieux), ainsi que de quelques galets de silex. L'ensemble reposait sur un horizon limoneux particulièrement plan. Les altitudes relevées à la base des pierres oscillaient entre 20,05 et 19,91 m NGF. Il est important de souligner que les bords dessinaient des limites franches et que la couche limono-sableuse ne renfermait pas d'autres roches de cette nature dans un large périmètre. Il ne semble donc pas s'agir d'un dépôt naturel, mais plutôt d'un aménagement anthropique, hypothèse qui tend à être confirmée par les résultats de l'examen ${ }^{1}$ d'une vingtaine de coquillages d'âge tertiaire qui étaient piégés sous plusieurs blocs et dans les interstices qui les séparaient.

Ce sont, en effet, 23 coquillages éocènes qui se trouvaient dispersés essentiellement dans la partie centrale de l'amas de roches. Bien que la plupart d'entre eux soient fragmentés, 12 éléments de la classe des gastéropodes, dont 7 exemplaires appartenant à la famille des Turritellidae et 5 à la famille des Potamididae, ainsi que 3 lamellibranches, dont 1 moule interne de bivalve, ont été identifiés. Les 8 autres fragments sont de trop petites dimensions pour permettre une détermination fiable.

Ce lot de coquillages fossiles paraît trop homogène pour que l'on puisse envisager qu'il se soit accumulé au sein de la structure pierreuse suite à un transfert naturel par le fleuve depuis un gîte fossilifère. Sa composition semble plutôt refléter un tri anthropique, notamment en faveur des morphologies turriculées. Aucun stigmate de perforation intentionnelle n'a été observé sur ces objets, mais des restes de matière colorante ont été repérés à fort grossissement sous la loupe binoculaire. En effet, bien que la surface des coquillages soit fortement lessivée, des traces évidentes (points et bandes) d'ocre rouge (ou d'hématite) subsistent à certains endroits protégés (cupules, fissures et espaces séparant les lamelles du test du coquillage). Ces traces résiduelles de coloration sont 

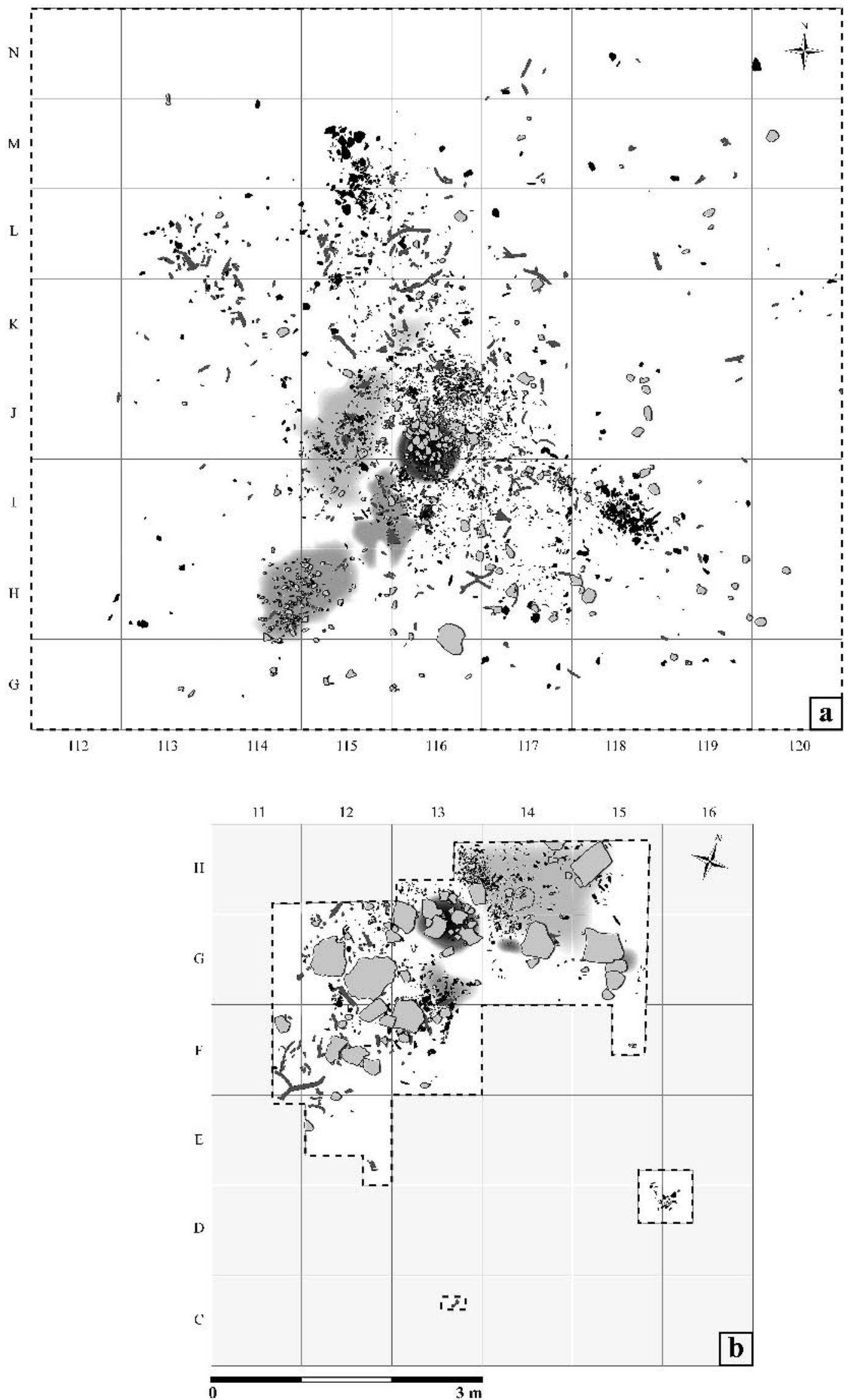

Fig. 20 - Plan de répartition des vestiges : a) unité J116 du niveau IV40 de Pincevent; b) unité G13 de la Haye.

Fig. 20 - Distribution maps of the Magdalenian horizon: a) occupation unit J116 of level IV40 at Pincevent; unit G13 at La Haye. 


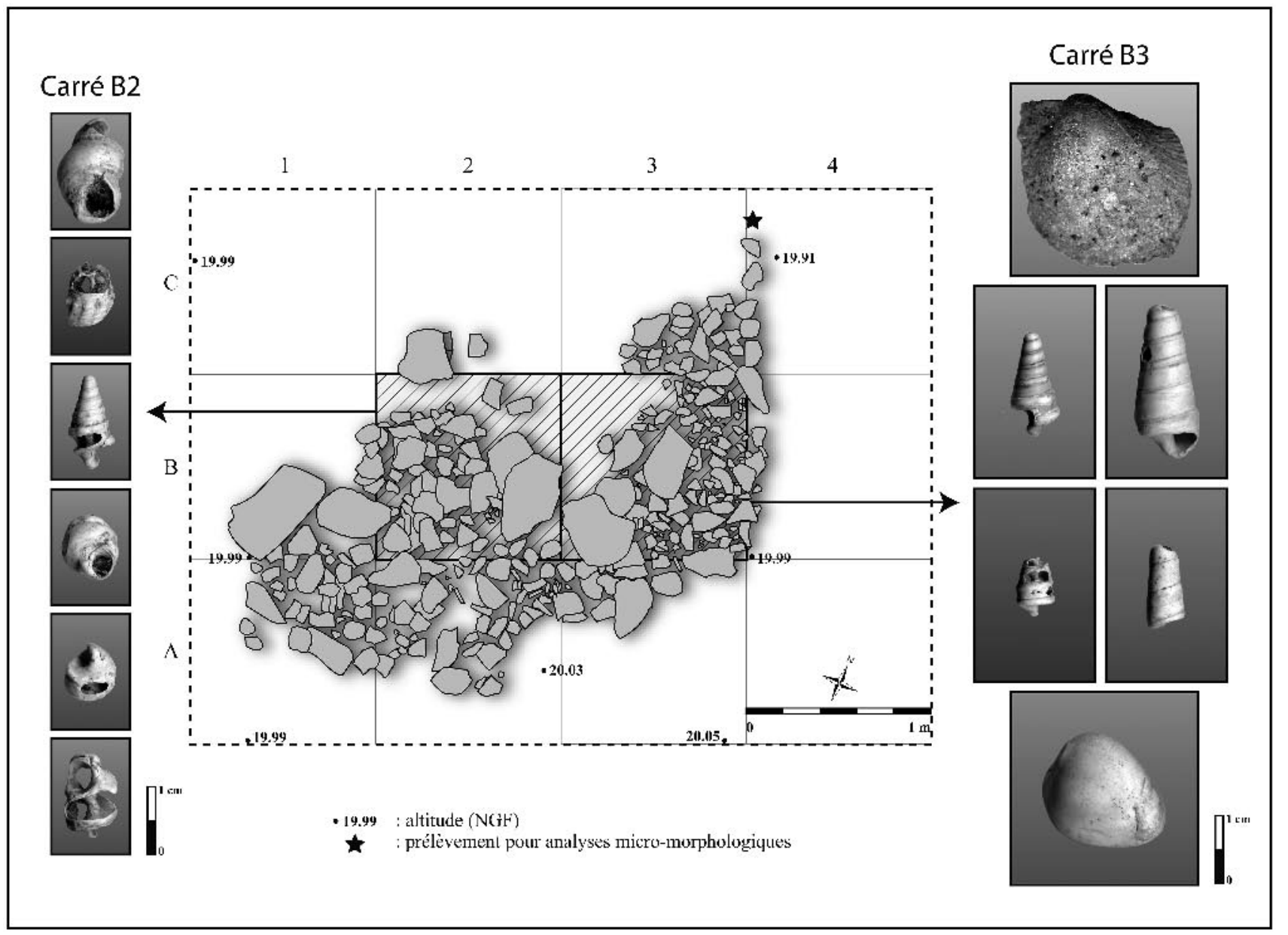

Fig. 21 - Plan de la structure pierreuse de la zone ouest.

Fig. 21 - Plan of the stone structure found in the western zone.

attestées sur 10 des 23 coquillages et constituent probablement le meilleur témoignage dont nous disposons actuellement pour attribuer à cet amas de roches une origine anthropique.

Tenter dès à présent de proposer une fonction à cette structure, c'est risquer de se perdre en conjectures. À notre connaissance, il n'existe aucune découverte du même type dans les gisements tardiglaciaires de plein air du Bassin parisien ou des régions voisines qui nous permettrait d'établir des comparaisons. Aucun des nombreux sondages réalisés à proximité n'a livré des vestiges susceptibles de nourrir la discussion. Aucun lien entre cet amas de roches et le riche niveau d'occupation dégagé à 500 mètres ne peut être démontré, mais cela doit néanmoins être envisagé, notamment parce que ce dernier niveau intègre également divers blocs de calcaire et de grès dans l'aménagement du lieu d'habitat et qu'il a livré un coquillage fossile de la famille des Turritellidae empreint d'une matière colorante. L'analyse des prélèvements de sédiment réalisés au sein de cette accumulation de roches fournira peutêtre des informations supplémentaires qui permettront d'aller plus loin dans l'interprétation. De même, l'analyse micromorphologique d'une petite berme prélevée en bordure immédiate de l'amas (fig. 21) devrait apporter des éléments concernant le degré d'anthropisation du sol sur lequel il reposait.

\section{MAGDALÉNIENS DES YVELINES : COMMENTAIRES SUR QUELQUES CONTRASTES RELEVÉS ENTRE LES GISEMENTS DE LA HAYE ET DE LA CÔTE-MASSET}

Le gisement de la Haye est localisé dans une aire géographique du Bassin parisien où les indices d'occupations magdaléniennes demeuraient ténus (fig. 22). Nous l'avons d'ailleurs constaté au travers des paragraphes précédents, c'est avec les gisements de plein air situés dans d'autres départements d'Île-de-France et de ses marges, tels que Pincevent (Seine-et-Marne), Étiolles (Essonne) et Verberie (Oise), que l'on peut établir les meilleures comparaisons.

Les environs immédiats de la Haye ont surtout livré des indices rattachés aux périodes du Paléolithique ancien et moyen. Divers vestiges ont été ramassés lors la surveillance des travaux d'exploitation des sables et des briqueteries (Lécolle, 1989; Carité, 2007). Les gisements de Bazemont et d'Herbeville constituent les seuls indices solides d'occupations pour le Paléolithique 
supérieur (à l'Aurignacien notamment; Bodu, 2008) situés à moins de 10 kilomètres de la Haye. Le rayon de recherche doit être étendu à environ 25 kilomètres pour rencontrer les premiers témoignages magdaléniens. Mentionnons tout d'abord la Butte-de-Beauregard à Clairefontaine-en-Yvelines qui a livré une petite quantité de silex parmi lesquels on recense des outils et des armatures caractéristiques du Magdalénien supérieur (Schmider, 1971). C'est à la faveur d'une opération de fouille préventive qu'a été ensuite découvert le gisement de plein air du Moulin-de-Lettrée à Neauphle-le-Vieux, au sein duquel fut mis au jour un amas de débitage conservé sur quelques mètres carrés et accompagné de quelques nucléus et outils sur lames (Giligny dir., 1997). Enfin, l'abri-sous-roche de la Côte-Masset à Bonnièressur-Seine conserve un ensemble de vestiges composés de quelques restes de faune, de déchets de taille, d'outils et d'armatures (Barois-Basquin et al., 1996). À l'échelle du département, la Côte-Masset constitue l'unique occupation magdalénienne qui a livré suffisamment de matériel pour permettre d'établir quelques comparaisons avec la Haye. Nous avons donc récemment réexaminé cet assemblage relativement abondant. La nature et la diversité de l'outillage sont caractéristiques du Magdalénien supérieur régional. Les remontages réalisés attestent qu'il en va de même en ce qui concerne les méthodes mises en œuvre pour produire les lames et les lamelles. Il faut toutefois évoquer la présence de quelques objets un peu particuliers : plusieurs becs à rostre très dégagé et une pointe à cran. Pour l'heure, nous ne disposons pas d'indice attestant de leur présence parmi les vestiges mis au jour à la Haye. Ils sont en revanche présents dans quelques autres industries magdaléniennes régionales que B. Schmider inscrit au sein d'un faciès spécifique (Schmider dir., 1992).

Les contrastes enregistrés dans le domaine de la faune chassée ont davantage retenu notre attention. Au cours des travaux de terrain réalisés en 1910 par A.-G. Poulain, des restes de cheval et de sanglier ainsi qu'une mâchoire de mégacéros avaient été découverts en association avec les nombreux objets en silex dans l'abri de la CôteMasset (Habasque et al., 1992). La reprise des opérations de fouilles en 1991 a permis de retrouver quelques éléments supplémentaires parmi lesquels une crache de Cervus elaphus, dont on a pu récemment déterminer qu'elle appartenait à un individu femelle de moins de deux ans, ainsi que diverses esquilles osseuses (c'est à partir de l'une d'entre elles qu'a été obtenue la date radiocarbone qui figure dans le tableau 1). Les quelques restes de faune provenant des déblais de la fouille de 1910 sont en cours d'étude. Les premiers résultats indiquent la présence de cerf, de chevreuil, de sanglier auxquels s'ajoutent quelques éléments du genre Canis, sans que l'on puisse pour le moment préciser l'espèce. C'est donc un tableau de chasse plutôt inhabituel que livre la Côte-Masset en regard de tous les autres sites régionaux. Si le cheval constitue, comme le renne, un taxon ubiquiste dans les occupations magdaléniennes du Bassin parisien (Bignon, 2007), les trois autres ne sont pas représentés, à l'exception de quelques restes de cerf identifiés au Pré-des-Forges à Marsangy (Poplin in Schmider dir., 1992) et au Brassot à Étigny (Bemilli in

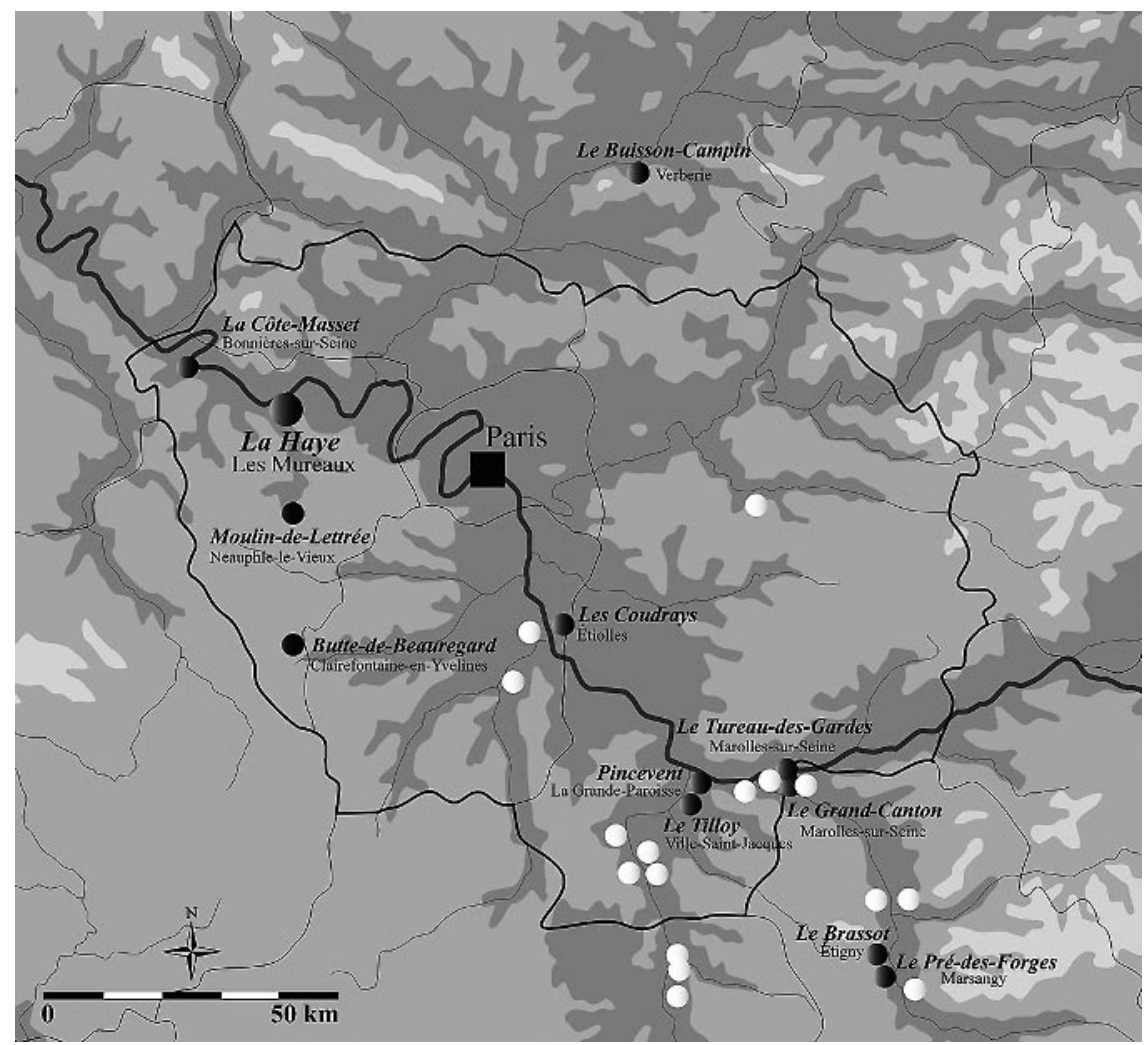

Fig. 22 - Répartition des gisements magdaléniens en Île-de-France et dans ses marges (point gris : sites mentionnés dans le texte).

Fig. 22 - Magdalenian sites in and around the Île-de-France region (grey dot: sites mentioned in the text). 
Connet et al., 1999). Dans sa globalité, le spectre de faune de la Côte-Masset se rapproche davantage de ceux des gisements magdaléniens de la moitié méridionale de la France (Costamagno, 1999). Plus au nord, en Belgique, les occupations magdaléniennes et creswelliennes du nord-ouest des Ardennes livrent aussi de bonnes correspondances (Cordy, 1991; Charles, 1998), même si des soupçons de contamination par des éléments plus récents ont été parfois évoqués pour expliquer la présence de restes de sanglier (Charles, 1998, p. 25). Il pourrait être intéressant d'élargir la discussion et les comparaisons avec les spectres de la faune chassée par les groupes de l'Azilien ancien, notamment celui du Closeau (Hauts-de-Seine) dont les dates radiocarbone sont très proches de celles obtenues pour les occupations magdaléniennes régionales et dans la mesure où il réunit également le cheval, le cerf, le sanglier mais aussi des éléments de Canis (Bémilli, 2000).

Nous achèverons ces quelques lignes consacrées à l'abri de la Côte-Masset en soulignant l'importance particulière que revêt la mâchoire de mégacéros. Les études récentes indiquent que ce taxon a recolonisé l'Europe au cours du Tardiglaciaire après avoir totalement disparu de ce vaste espace durant le Dernier Maximum glaciaire (Barnosky, 1986; Aaris-Sørensen et Liljegren, 2004; Stuart et al., 2004). Jusqu'à présent, les découvertes, exclusivement réalisées dans des contextes non anthropiques (dans les lacs et marais notamment), se cantonnaient à la Grande-Bretagne, à l'Irlande et à la Scandinavie. La plus ancienne date ${ }^{14} \mathrm{C}$ obtenue sur ce taxon, calculée à partir de restes provenant de l'île de Man, en mer d'Irlande, est de $12455 \pm 65$ BP (Stuart et al., 2004). À notre connaissance, la mâchoire de mégacéros de la Côte-Masset constitue non seulement le premier témoin d'âge tardiglaciaire pour le nord de la France, mais aussi l'unique reste dans toute l'Europe septentrionale recueilli dans un gisement archéologique.

\section{PERSPECTIVES}

L'état de conservation remarquable du sol d'habitat magdalénien de la Haye confère à ce gisement un statut particulier. D'ores et déjà, il s'inscrit parmi les sites de référence de cette période qui permettent de mener une analyse palethnographique fine. En outre, l'interstratification du sol d'habitat dans une puissante séquence sédimentaire permettra sans doute de préciser le calage chronologique de l'occupation et, peut-être, de définir la chronozone du Tardiglaciaire au cours de laquelle se développent les épaisses couches d'alluvions limono-sableuses qui contiennent les occupations magdaléniennes de fond de vallée en Île-de-France.

L'analyse, à peine esquissée ici, ne pourra évidemment être complétée que si ce sol d'habitat fait l'objet d'une fouille exhaustive. Le projet de construction du circuit automobile ayant été abandonné, aucune menace immédiate ne pèse sur ce site. On ne peut toutefois garantir la protection définitive du gisement dans la mesure où les terrains se trouvent en zone constructible. De plus, comme l'indique l'étude géoarchéologique, il existe un fort potentiel vers l'est, au-delà de la surface diagnostiquée, sur une superficie de plus de neuf hectares. Il s'agit donc d'un secteur sous haute surveillance archéologique dans lequel d'autres sondages devront être pratiqués afin d'en évaluer précisément la richesse archéologique et de prendre les mesures adaptées à sa sauvegarde.

Remerciements : Il nous est agréable de remercier nos collègues des UMR 7041 et 7055 venus nous apporter leur précieux soutien sur le terrain et pour l'intérêt qu'ils ont porté à la découverte du gisement : F. Audouze, P. Bodu, M. Christensen, M. Julien, D. Legoupil, M. Olive, M. Orliac, J. Pelegrin, B. Valentin, M. Vanhaeren. Nos remerciements s'adressent aussi C. KuharSiffert et S. Païn (service archéologique départemental des Yvelines) pour leur aide dans le traitement et la conservation des vestiges, à D. Copel, conservatrice du musée A.-G.-Poulain de Vernon, pour nous avoir autorisés à réexaminer la collection de la Côte-Masset, ainsi qu'à M. Pionnier et J.-D. Vigne pour leur aide à l'identification des restes de faune. Un grand merci aussi à D. Leesch (Laboratoire d'archéozoologie de l'université de Neuchâtel) pour l'ensemble de ses conseils et relectures.

\section{NOTE}

(1) Nous adressons nos plus vifs remerciements à M. Christensen (université Paris 1-Panthéon Sorbonne et CNRS, UMR 7041, équipe Ethnologie préhistorique) qui a mis à notre disposition le matériel nécessaire pour réaliser les observations à fort grossissement et les prises de vue présentées en figure 21 .

\section{RÉFÉRENCES BIBLIOGRAPHIQUES}

AARIS-SøRENSEN K., LILJEGREN R. (2004) - Late Pleistocene Remains of Giant Deer (Megaloceros giganteus Blumenbach) in Scandinavia: Chronology and Environment, Boreas, 33, p. 61-73.

ANTOINE P., LIMONDIN-LOZOUET N., CHAUSSÉ C., LAUTRIDOU J.P., PASTRE J.F., AUGUSTE P., BAHAIN J.J., FALGUÈRES C., GALEHB B. (2007) - Pleistocene Fluvial Terraces from Northern France (Seine, Yonne, Somme): Synthesis and New Results, Quaternary Science Reviews, 26, p. 2701-2723.

AUDOUZE F., KARLIN C., CAHEN D., CROISSET E. DE, COUDRET P., LARRIERE M., MASSON P., MAUGER M., OLIVE M., PELEGRIN J., PIGEOT N., PLISSON H., SCHMIDER B., TABORIN Y. (1988) - Taille du silex et finalité du débitage dans le
Magdalénien du Bassin parisien, in M. Otte dir., De la Loire à l'Oder : les civilisations du Paléolithique final dans le nord-ouest européen, t. 1, Actes du colloque international de Liège, décembre 1985, Éd. Université de Liège-BAR (ERAUL 25- BAR International Series 444), p. $55-84$.

BARNOSKY A.D. (1986) - "Big Game" Extinction Caused by Late Pleistocene Climatic Change: Irish Elk (Megaloceros giganteus) in Ireland, Quaternary Research, 25, p. 128-135.

BAROIS-BASQUIN B., CHARIER M.-A., LÉCOLLE F. (1996) - Un abri-sous-roche de la fin du Paléolithique supérieur à Bonnières-surSeine (Yvelines), Bulletin de la Société préhistorique française, 93, 1, p. 33-42. 
BÉMILLI C. (2000) - Nouvelles données sur les faunes aziliennes du Closeau, Rueil-Malmaison (Hauts-de-Seine), in G. Pion dir., Le Paléolithique supérieur récent : nouvelles données sur le peuplement et l'environnement, Actes de la table ronde de Chambéry, mars 1999 Paris, Éd. Société préhistorique française (Mémoire 28), p. 29-38.

BIGNON O. (2007) - L'autre «civilisation du Renne»... pour une réinterprétation des stratégies cynégétiques au Magdalénien dans le Bassin parisien, in S. Beyries, D. Drucker et V. Vaté dir., Les civilisations du renne d'hier et d'aujourd'hui : approches ethnohistoriques, archéologiques et anthropologiques, Actes des $27^{\mathrm{es}}$ Rencontres internationales d'archéologie et d'histoire d'Antibes, octobre 2006, Antibes, Éd. APDCA, p. 223-241.

BODU P. (1993) - Analyse typotechnologique du matériel lithique de quelques unités du site magdalénien de Pincevent (Seine-et-Marne) applications spatiales, économiques et sociales, Thèse de doctorat, Université Paris 1-Panthéon Sorbonne, Paris, 3 vol., 852 p.

BODU P. dir. (1998) - Le Closeau : deux années de fouille sur un gisement azilien et belloisien en bord de Seine, Rueil-Malmaison (Hauts de-Seine), Document final de synthèse de sauvetage urgent, AFAN, Saint-Denis, SRA d'Île-de-France, 3 vol., 470 p.

BODU P. (2008) - Le Paléolithique supérieur dans les Yvelines, in F. Giligny dir., La préhistoire en val de Seine, Catalogue d'exposition, Musée de 1'Hôtel-Dieu, Mantes-la-Jolie, 2008-2009, Paris, Éd. Somogy éditions d'art, p. 24-33.

BODU P., DEBOUT G., TABORIN Y. (2005) - De la parure chez les Badegouliens du Bassin parisien : le cas du site de Oisy dans la Nièvre, in V. Dujardin dir., Industrie osseuse et parures du Solutréen au Magdalénien en Europe, Paris, Éd. Société préhistorique française (Mémoire 39), p. 87-99.

BODU P., DEBOUT G., LEESCH D., SCHOCH W., VALENTIN B. (2008) - Révision de la chronologie magdalénienne à Pincevent : l'apport des micro-charbons de bois, in B. Valentin dir., Habitats et peuplements tardiglaciaires du Bassin parisien, Rapport de projet collectif de recherche, Nanterre - Saint-Denis, UMR 7041-SRA d'Îlede-France, p. 53-63.

BODU P., DEBOUT G., DUMARÇAY G., LEESCH D., VALENTIN B. (2009) - Révision de la chronologie magdalénienne dans le Bassin parisien et alentours : nouveaux résultats, in B. Valentin dir., Habitats et peuplements tardiglaciaires du Bassin parisien, Rapport de projet collectif de recherche, Nanterre - Saint-Denis, UMR 7041-SRA d'Îlede-France, p. 91-107.

CARITÉ D. (2007) - Un site de type acheuléen dans la plaine alluviale à Flins-sur-Seine, Bulletin du Centre de recherches archéologiques de la région mantaise, 17, p. 19-26.

CHABROL A., CHRISTENSEN M., OLIVE M., ROBLIN-JOUVE A., RODRIGUEZ P., SAMZUN A. (2008) - Rive droite, rive gauche : les occupations magdaléniennes d'Étiolles (Essonne), Revue archéologique d'Île-de-France, 1, p. 7-20.

CHARLES R. (1998) - Late Magdalenian Chronology and Faunal Exploitation in the North-Western Ardennes, Oxford, Éd. Archaeopress (BAR International Series 737), 246 p.

CONNET N., LHOMME V., ALLENET G., BÉMILLI C., CHAUSSÉ S., LEROYER C., LIMONDIN N. (1999) - Le gisement du Paléolithique supérieur final du Brassot à Étigny (Yonne), Document final de synthèse de sauvetage urgent, AFAN, Dijon, SRA de Bourgogne, 117 p.

CORDY J.-M. (1991) - Palaeoecology of the Late Glacial and early Postglacial of Belgium and Neighbouring Areas, in N. Barton, A.J. Roberts and D.A. Roe dir., The Late Glacial in North-West Europe: Human Adaptation and Environmental Change at the End of the Pleistocene, York, Éd. CBA (Research Report 77), p. 40-47.

COSTAMAGNO S. (1999) - Stratégies de chasse et fonction des sites au Magdalénien dans le sud de la France, Thèse de doctorat, Université Bordeaux 1, Talence, 495 p.

DAVID F. (1994) - La faune de mammifères de Pincevent et Verberie, in Y. Taborin dir., Environnements et habitats magdaléniens dans le centre du Bassin parisien, Paris, Éd. Maison des sciences de l'homme (Documents d'archéologie française 43), p. 105-110.

DEBOUT G. (2003) - Les microlithes du Magdalénien supérieur dans le Bassin parisien : une diversité inattendue, in É. Ladier dir., Les pointes à cran dans les industries lithiques du Paléolithique supérieur récent de l'oscillation de Lascaux à l'oscillation de Bölling, Actes de la table ronde de Montauban, avril 2002, Cressensac, Éd. Association préhistoire du Sud-Ouest (Préhistoire du Sud-Ouest supplément 6), p. 91-100.

DEBOUT G. (2007) - Un atelier de peausserie il y a 12000 ans à Pincevent?, in S. Beyries, D. Drucker et V. Vaté dir., Les civilisations du renne d'hier et d'aujourd'hui : approches ethnohistoriques, archéologiques et anthropologiques, Actes des $27^{\text {es }}$ Rencontres internationales d'archéologie et d'histoire d'Antibes, octobre 2006, Antibes, Éd. APDCA, p. 439-455.

DEBOUT G. dir. (2009) - Flins-sur-Seine - Les Mureaux (Yvelines), "Vallée de l'automobile et de la mobilité durable», Rapport de diagnostic, Conseil général des Yvelines, Saint-Denis, SRA d'Île-deFrance, 2 vol., 695 p.

GILIGNY F. dir. (1997) - Les occupations pré- et protohistorique du vallon de la Guyonne, Neauphle-le-Vieux, le Moulin-de-Lettrée, Yvelines, Rapport de fouille, AFAN, Saint-Denis, SRA d'Île-de-France, 2 vol., $386 \mathrm{p}$.

GRIMM S.B., WEBER M.-J. (2008) - The Chronological Framework of the Hamburgian in the Light of Ild and New ${ }^{14} \mathrm{C}$ Dates, Quartär, 55 , p. $17-40$.

HABASQUE G., CHARIER M.-A., BAROIS-BASQUIN B., LÉCOLLE F. (1992) - Bonnières-sur-Seine : l'abri sous roche de la Côte-Masset (Yvelines), Rapport de fouille, Versailles, Service archéologique départemental des Yvelines, $99 \mathrm{p}$.

JACOBI R. (1991) - The Creswellian, Creswell and Cheddar, in N. Barton, A.J. Roberts and D.A. Roe dir., The Late Glacial in North-West Europe: Human Adaptation and Environmental Change at the End of the Pleistocene, York, Éd. CBA (Research Report 77), p. 128-140.

JULIEN M (1988) - Organisation de l'espace et fonction des habitats magdaléniens du Bassin parisien, in M. Otte dir., De la Loire à l'Oder: les civilisations du Paléolithique final dans le nord-ouest européen, t. 1, Actes du colloque international de Liège, décembre 1985, Éd. Université de Liège-BAR (ERAUL 25- BAR International Series 444), p. 81-123.

JULIEN M., KARLIN C. (2002) - Un habitat saisonnier de plein air au Tardiglaciaire : l'exemple d'un campement à Pincevent, Seine-et-Marne, in J.-C. Miskovsky dir., Géologie de la Préhistoire, p. 1399-1410.

JULIEN M., BEYRIES S. (2006) - Sur quelques objets mobiliers domestiques, in P. Bodu, M. Julien, B. Valentin et G. Debout dir., Un dernier hiver à Pincevent : les Magdaléniens du niveau IV-O, Gallia Préhistoire, 48, p. 79-83.

LE JEUNE Y., PASTRE J.-F., ARCAY D., AUBRY L., CAMERLYNCK C., CONFALONIERI J., LANCHON Y., VERGNAUD D., VISSAC C. (2005) - Variabilité de l'enregistrement alluvial holocène ; l'exemple du méandre de la Haute-Île (vallée de la Marne, bassin parisien), premiers résultats, Quaternaire, 16, 4, p. 299-313.

LÉCOLLE F. (1989) - Le cours moyen de la Seine au Pléistocène moyen et supérieur: géologie et préhistoire, Caen, Éd. Centre de géomorphologie du CNRS-Groupe Seine, 549 p.

LEROI-GOURHAN A., BREZILLON M. (1972) - Fouilles de Pincevent : essai d'analyse ethnographique d'un habitat magdalénien (la section 36), Paris, Éd. CNRS (Supplément à Gallia Préhistoire 7), 345 p.

LEROYER C. (1994) - Le paysage végétal au Tardiglaciaire : apport de la palynologie, in Y. Taborin dir., Environnements et habitats magdaléniens dans le centre du Bassin parisien, Paris, Éd. Maison des sciences de l'homme (Documents d'archéologie française 43), p. 59-64.

MARLON J.R., BARTLEIN P.J., WALSH M.K., HARRISON S.P. BROWN K.J., EDWARDS M.E., HIGUERA P.E., POWER M.J., ANDERSON R.S., BRILES C., BRUNELLE A., CARCAILLET C., DANIELS M., HU F.S., LAVOIE M., LONG C., MINCKLEY T., RICHARD P.J.H., SCOTT A.C., SHAFER D.S., TINNER W. UMBANHOWAR C.E. Jr, WHITLOCK C. (2009) - Wildfire Responses to Abrupt Climate Change in North America, Proceedings of the National Academy of Sciences, 106, 8, p. 2519-2524.

MAUGER M. (1994) - L'approvisionnement en matériaux siliceux au Paléolithique supérieur, in Y. Taborin dir., Environnements et habitats magdaléniens dans le centre du Bassin parisien, Paris, Éd. Maison des sciences de l'homme (Documents d'archéologie française 43), p. 78-93. 
OLIVE M., AUDOUZE F., JULIEN M. (2000) - Nouvelles données concernant les campements magdaléniens du Bassin parisien, in B. Valentin, P. Bodu et M. Christensen dir., L'Europe centrale et septentrionale au Tardiglaciaire : confrontation des modèles régionaux de peuplement, Éd. APRAIF (Mémoire du musée de Préhistoire d'Île-de-France 7), Nemours, p. 289-304.

ORLIAC M. (2006) - Position stratigraphique du niveau IV-0, in P. Bodu, M. Julien, B. Valentin et G. Debout dir., Un dernier hiver à Pincevent : les Magdaléniens du niveau IV-0, Gallia Préhistoire, 48, p. 8-15.

PASTRE J.-F., FONTUGNE M., KUZUCUOGLU C., LEROYER C., LIMONDIN-LOZOUET N., TALON M., TISNERAT N. (1997)L'évolution tardi- et postglaciaire des lits fluviaux au nord-est de Paris (France) : relations avec les données paléoenvironnementales et l'impact anthropique sur les versants, Géomorphologie, relief, processus, environnement, 4, p. 291-312.

PASTRE J.-F., LEROYER C., LIMONDIN-LOZOUET N., CHAUSSÉ C., FONTUGNE M., GEBHARDT A., HATTE C., KRIER V. (2000) - Le tardiglaciaire des fonds de vallée du Bassin parisien (France), Quaternaire, 11, 2, p. 107-122.

PASTRE J.-F., LEROYER C., LIMONDIN-LOZOUET N., ORTH P., CHAUSSÉ C., FONTUGNE M., GAUTHIER A, KUNESH S., LE JEUNE Y., SAAD M.-C. (2002) - Variations paléoenvironnementales et paléohydrologiques durant les 15000 derniers millénaires : les réponses morphosédimentaires des vallées du Bassin parisien, in J.-P. Bravard et M. Magny dir., Les fleuves ont une histoire : paléoenvironnement des rivières et des lacs français depuis 15000 ans, Paris, Éd. Errance, p. 29-44.

PASTRE J.-F., LIMONDIN-LOZOUET N., LEROYER C., PONEL Ph., FONTUGNE M. (2003) - River System Evolution and Environmental Changes During the Lateglacial in the Paris Basin (France), Quaternary Science Reviews, 22, p. 2177-2188.

PÉTILLON J.-M. (2008) - Spécificités des armatures osseuses magdaléniennes du Bassin parisien, in B. Valentin dir., Habitats et peuplements tardiglaciaires du Bassin parisien, Rapport de projet collectif de recherche, Nanterre - Saint-Denis, UMR 7041-SRA d'Île-deFrance, p. 25-30.

RASMUSSEN S.O., ANDERSEN K.K., SVENSSON A.M., STEFFENSEN J.P., VINTHER B.M., CLAUSEN H.B., SIGGAARD ANDER SEN M.-L., JOHNSEN S.J., LARSEN L.B., DAHL-JENSEN D., BIGLER M., RÖTHLISBERGER R., FISCHER H., GOTO-AZUMA K., HANSSON M.E., RUTH U. (2006) - A New Greenland Ice Core Chronology for the Last Glacial Termination, Journal of Geophysical Research 111, D06102, doi:10.1029/2005JD006079.

ROBLIN-JOUVE A. (1994) - Le milieu physique, in Y. Taborin dir., Environnements et habitats magdaléniens dans le centre du Bassin parisien, Paris, Éd. Maison des sciences de l'homme (Documents d'archéologie française 43), p. 12-35.

SCHMIDER B. (1971) - Les industries lithiques du Paléolithique supérieur en Île-de-France, Paris, Éd. CNRS (Supplément à Gallia Préhistoire 6), $243 \mathrm{p}$.

SCHMIDER B. dir. (1992) - Marsangy : un campement des derniers chasseurs magdaléniens, sur les bords de l'Yonne, Liège, Ed. Université de Liège (ERAUL 55), 275 p.

STUART A.J., KOSINTSEV P.A., HIGHAM T.F.G., LISTER A.M (2004) - Pleistocene to Holocene Extinction Dynamics in Giant Deer and Woolly Mammoth, Nature, 431, p. 684-689.

TABORIN Y. (1994) - Les coquillages marins, in Y. Taborin dir., Environnements et habitats magdaléniens dans le centre du Bassin parisien, Paris, Éd. Maison des sciences de l'homme (Documents d'archéologie française 43), p. 70-77.

VALENTIN B. (1995) - Les groupes humains et leurs traditions au Tardiglaciaire dans le Bassin parisien : apports de la technologie comparée, Thèse de doctorat, Université Paris 1-Panthéon Sorbonne, Paris, 3 vol., 1106 p.

VALENTIN B. (2008) - Jalons pour une paléohistoire des dernier chasseurs (XIV $-V I^{e}$ millénaires avant J.-C.), Paris, Éd. Publications de la Sorbonne, Paris, $325 \mathrm{p}$.

VALENTIN B., PIGEOT N. (2000) - Éléments pour une chronologie des occupations magdaléniennes dans le Bassin parisien, in B. Valentin,
P. Bodu et M. Christensen dir., L'Europe centrale et septentrionale au Tardiglaciaire: confrontation des modèles régionaux de peuplement, Nemours, Éd. APRAIF (Mémoire du musée de Préhistoire d'Île-de-France 7), p. 129-138.

VANHAEREN M. (2006) - La parure : de sa production à la projection de l'image de soi, in P. Bodu, M. Julien, B. Valentin et G. Debout dir., Un dernier hiver à Pincevent: les Magdaléniens du niveau IV-O, Gallia Préhistoire, 48, p. 35-49.

Grégory DEBOUT

Conseil général des Yvelines Service archéologique départemental

Le Pas-du-Lac, 2, avenue de Lunca 78180 Montigny-le-Bretonneux et Université Paris-Ouest - Nanterre-la Défense -

CNRS, ARSCAN UMR 7041 Équipe Ethnologie préhistorique gdebout@yvelines.fr

Yann LE JEUNE

Service régional de l'Archéologie

DRAC des Pays de la Loire

1, rue Stanislas-Baudry, BP 63518, 44035 Nantes cedex 1 et CNRS, Laboratoire de géographie physique UMR 8591, équipe Environnements quaternaires continentaux, dynamiques naturelles et anthropisation yann.le-jeune@culture.gouv.fr

Hélène DJEMA

Conseil général des Yvelines Service archéologique départemental

Le Pas-du-Lac, 2, avenue de Lunca 78180 Montigny-le-Bretonneux et Université Paris-Ouest - Nanterre-la Défense -

CNRS, ARSCAN UMR 7041 Équipe Ethnologie préhistorique hdjema@yvelines.fr

Olivier BIGNON

Université Paris-Ouest - Nanterre-la Défense CNRS, ARSCAN UMR 7041

Équipe Ethnologie préhistorique Maison d'archéologie et d'ethnologie 21, allée de l'Université, 92023 Nanterre cedex olivier.bignon@mae.u-paris10.fr

Marie-Aline CHARIER

Conseil général des Yvelines Service archéologique départemental Le Pas-du-Lac, 2, avenue de Lunca 78180 Montigny-le-Bretonneux macharier@yvelines.fr

Caroline PESCHAUX

Université Paris-Ouest - Nanterre-la Défense CNRS, ARSCAN UMR 7041

Équipe Ethnologie préhistorique Maison d'archéologie et d'ethnologie 21, allée de l'Université, 92023 Nanterre cedex et Université Paris 1-Panthéon Sorbonne Caroline.Peschaux@malix.univ-paris1.fr 